\title{
Spherical alterations of handles: embedding the manifold plus construction
}

\author{
Craig R Guilbault \\ FREDERICK C TINSLEY
}

\begin{abstract}
Quillen's famous plus construction plays an important role in many aspects of manifold topology. In our own work [4] on ends of open manifolds, an ability to embed cobordisms provided by the plus construction into the manifolds being studied was a key to completing the main structure theorem. In this paper we develop a "spherical modification" trick that allows for a constructive approach to obtaining those embeddings. More importantly, this approach can be used to obtain more general embedding results. In this paper we develop generalizations of the plus construction (together with the corresponding group-theoretic notions) and show how those cobordisms can be embedded in manifolds satisfying appropriate fundamental group properties. Results obtained here are motivated by, and play an important role in, our ongoing study of noncompact manifolds [2].
\end{abstract}

57N15, 57Q12; 57Q10, 57R65

\section{Introduction}

In this paper we develop a procedure, called "spherical alteration", for modifying handle decompositions of manifolds in ways that permit useful applications. The strategy is geometrically quite simple, but at the same time more drastic than the traditional techniques of handle slides, introductions and cancellations of complementary handle pairs, and the carving out and inserting of existing handles. In order to obtain the intended applications, each alteration of a handle is accompanied by associated alterations of related submanifolds. Taken together, these moves constitute the process of spherical alteration. Since there are several variables involved, a full description of the procedure is a bit technical; we save that for Section 3. In some sense, our main result is more a technique than a specific theorem; nevertheless, several concrete applications of that technique are provided. The prototypical application is a constructive proof of the following theorem, which was a key ingredient in the main result in our work [4]. 
Theorem 1.1 (Embedded manifold plus construction) Let $R$ be a connected manifold of dimension $\geq 6, B$ be a closed component of $\partial R$, and

$$
K \subseteq \operatorname{ker}\left(\pi_{1}(B) \rightarrow \pi_{1}(R)\right)
$$

a perfect group that is the normal closure in $\pi_{1}(B)$ of a finite set of elements. Then there exists an embedding of a plus cobordism $(W, A, B)$ into $R$ which is the identity on $B$ and for which $\operatorname{ker}\left(\pi_{1}(B) \rightarrow \pi_{1}(W)\right)=K$.

Remark 1 (a) Recall that compact cobordism $(W, A, B)$ is a plus cobordism if $A \hookrightarrow W$ is a simple homotopy equivalence. A detailed discussion of plus cobordisms and the manifold plus construction can be found in Section 4.

(b) As an added bonus, our proof of Theorem 1.1 provides a new twist on the existence proof for plus cobordisms; an argument that requires very little discussion of bundles and framings.

We will further exhibit the usefulness of the spherical alteration technique by proving a generalization of Theorem 1.1. That generalization is motivated by ongoing work on ends of noncompact manifolds. It and a similar application of spherical alteration, also presented here, play key roles in our forthcoming paper [2]. To the best of our knowledge, these latter two applications are not obtainable by the nonconstructive approach to Theorem 1.1 used in [4].

Acknowledgement This project was aided by a Simons Foundation Collaboration Grant awarded to the first author.

\section{Preliminaries}

In this section we provide brief reviews of several topics and introduce a good deal of notation to be used later in this paper. Those topics are:

- Intersection numbers between submanifolds

- Surgering surfaces to disks and 2-spheres

- Perfect groups and "near perfect" subgroups

- Basics of handle theory

- Unbased spheres as elements of homotopy groups

Throughout this paper we work in the category of piecewise-linear manifolds; analogous results in the smooth and topological categories may be obtained in the usual ways. 


\subsection{Intersection numbers}

One of the simplest types of intersection number is defined when $P^{p}$ and $Q^{q}$ are closed, connected, oriented submanifolds of the interior of an oriented $(p+q)$-manifold $N$. First arrange that $P^{p}$ and $Q^{q}$ intersect transversely at a finite set of points $p_{1}, p_{2}, \ldots, p_{k}$. At each $p_{i}$, the local orientation of $P^{p}$ together with the local orientation of $Q^{q}$ (in that order) determine a local orientation for $N$. If that orientation agrees with the global orientation of $N$, we write $\operatorname{sgn}\left(p_{i}\right)=1$; otherwise $\operatorname{sgn}\left(p_{i}\right)=-1$. The $\mathbb{Z}$-intersection number is defined by $\varepsilon_{\mathbb{Z}}\left(P^{p}, Q^{q}\right)=\sum_{i=1}^{k} \operatorname{sgn}\left(p_{i}\right)$. This definition depends upon order; by linear algebra $\varepsilon_{\mathbb{Z}}\left(Q^{q}, P^{p}\right)=(-1)^{p q} \varepsilon_{\mathbb{Z}}\left(P^{p}, Q^{q}\right)$.

A more delicate intersection "number" lies in $\mathbb{Z}\left[\pi_{1}(N, *)\right]$. Instead of assuming $N$ is oriented (or even orientable), choose a local orientation of $N$ at $*$. Assume that $P^{p}$ and $Q^{q}$ are both oriented and simply connected, and fix base paths $\lambda_{P}$ and $\mu_{Q}$ in $N$ from $*$ to base points $*_{P} \in P^{p}$ and $*_{Q} \in Q^{q}$. For each $p_{i}$, choose paths $\rho_{i}$ in $P^{p}$ and $\sigma_{i}$ in $Q^{q}$ from the respective base points to $p_{i}$. Let $\operatorname{sgn}\left(p_{i}\right)= \pm 1$, depending on whether the local orientation at $*$ translated along the path $\lambda_{P} \cdot \rho_{i}$ agrees with orientation at $p_{i}$ induced by the orientations of $P^{p}$ then $Q^{q}$; then let $g_{i} \in \pi_{1}(N, *)$ correspond to $\lambda_{P} \cdot \rho_{i} \cdot \sigma_{i}^{-1} \cdot \mu_{Q}^{-1}$. At $p_{i}$ define $\varepsilon_{\mathbb{Z} \pi_{1}(N, *)}\left(p_{i}\right)=\operatorname{sgn}\left(p_{i}\right) g_{i}$. Finally, the $\mathbb{Z} \pi_{1}$-intersection number is defined by

$$
\varepsilon_{\mathbb{Z} \pi_{1}(N, *)}\left(P^{p}, Q^{q}\right)=\sum_{i=1}^{k} \varepsilon_{\mathbb{Z} \pi_{1}(N, *)}\left(p_{i}\right) \in \mathbb{Z}\left[\pi_{1}(N, *)\right] .
$$

Note that simple connectivity of $P^{p}$ and $Q^{q}$ ensures that $\varepsilon_{\mathbb{Z} \pi_{1}(N, *)}\left(P^{p}, Q^{q}\right)$ does not depend on the choice of $\rho_{i}$ and $\sigma_{i}$, however there is some dependence on $\lambda_{P}$ and $\mu_{Q}$. The ordering of $P^{p}$ and $Q^{q}$ now plays a larger role than it did for $\mathbb{Z}$-intersection numbers: a change in order first alters $\operatorname{sgn}\left(p_{i}\right)$ by a factor of $(-1)^{p q} \omega_{1}\left(g_{i}\right)$, where $\omega_{1}\left(g_{i}\right)=1$ if $g_{i}$ is an orientation preserving loop and $\omega_{1}\left(g_{i}\right)=-1$ otherwise; secondly, the loop $\lambda_{P} \cdot \rho_{i} \cdot \sigma_{i}^{-1} \cdot \mu_{Q}^{-1}$ is now traversed in the opposite direction, so $g_{i}$ becomes $g_{i}^{-1}$. For us, the key facts related to order are:

- $\varepsilon_{\mathbb{Z} \pi_{1}(N, *)}\left(P^{p}, Q^{q}\right)=0$ if and only if $\varepsilon_{\mathbb{Z} \pi_{1}(N, *)}\left(Q^{q}, P^{p}\right)=0$.

- If $\varepsilon_{\mathbb{Z} \pi_{1}(N, *)}\left(P^{p}, Q^{q}\right)=1$ then $\varepsilon_{\mathbb{Z} \pi_{1}(N, *)}\left(Q^{q}, P^{p}\right)= \pm 1$.

Sometimes the simple connectivity conditions on $P^{p}$ and $Q^{q}$ can be relaxed. An important such case occurs when one of the submanifolds, say $Q^{q}$, is a 1-sphere; there we salvage "well-definedness" by requiring that $\sigma_{i}$ be the unique arc of $Q^{q}$ running from $* Q$ to $p_{i}$ in the orientation preserving direction. Another useful variation occurs when the fundamental group of $P^{p}$ or $Q^{q}$ includes trivially into the that of $N$, in which case that submanifold need not be simply connected. Similarly, if the 
images of $\pi_{1}\left(P^{p}\right)$ and $\pi_{1}\left(Q^{q}\right)$ (translated appropriately along $\lambda_{P}$ and $\mu_{Q}$ ) lie in an $L \unlhd \pi_{1}(N, *)$ then the above procedure produces a well-defined intersection number in $\mathbb{Z}\left[\pi_{1}(N, *) / L\right]$.

We will call collections $\left\{P_{i}^{p}\right\}_{i=1}^{r}$ and $\left\{Q_{i}^{q}\right\}_{i=1}^{r}$ of closed submanifolds of a $(p+q)-$ manifold $N^{n}$ geometrically dual if $P_{i}^{p}$ intersects $Q_{i}^{q}$ transversely in a single point for all $i$ and $P_{i}^{p} \cap Q_{j}^{q}=\varnothing$ for all $i \neq j$. If $N^{n}$ and all of these submanifolds are oriented, then the collections are algebraically dual over $\mathbb{Z}$ if $\varepsilon_{\mathbb{Z}}\left(P_{i}^{p}, Q_{j}^{q}\right)= \pm \delta_{i j}$ for all $1 \leq i, j \leq r$. So (given the necessary orientability requirements), collections which are geometrically dual are necessarily algebraically dual over $\mathbb{Z}$, but not conversely.

More generally, given the necessary hypotheses and all required choices to make $\mathbb{Z}\left[\pi_{1}(N, *)\right]$-intersection numbers well-defined, collections $\left\{P_{i}^{p}\right\}_{i=1}^{r}$ and $\left\{Q_{i}^{q}\right\}_{i=1}^{r}$ are algebraically dual over $\mathbb{Z}\left[\pi_{1}(N, *)\right]$ if $\varepsilon_{\mathbb{Z}\left[\pi_{1}(N, *)\right]}\left(P_{i}^{p}, Q_{j}^{q}\right)= \pm \delta_{i j}$ for all $1 \leq$ $i, j \leq r$. In reality, we are usually satisfied if each $\varepsilon_{\mathbb{Z}\left[\pi_{1}(N, *)\right]}\left(P_{i}^{p}, Q_{i}^{q}\right)= \pm g_{i}$ for some $g_{i} \in \pi_{1}(N, *)$ and $\varepsilon_{\mathbb{Z}\left[\pi_{1}(N, *)\right]}\left(P_{i}^{p}, Q_{j}^{q}\right)=0$ when $i \neq j$. In those cases, we can always arrange the more rigid requirement by rechoosing some of the base paths. Under appropriate conditions, the notion of collections being algebraically dual over $\mathbb{Z}\left[\pi_{1}(N, *) / L\right]$ may be defined in a similar manner.

\subsection{Surgery on surfaces}

For a compact oriented surface $\Lambda$ with zero or one boundary components, a complete set of meridian-longitude pairs is a collection of pairs of oriented simple closed curves $\left\{\left(m_{j}, l_{j}\right)\right\}_{j=1}^{k}$ such that collections $\left\{m_{j}\right\}_{j=1}^{k}$ and $\left\{l_{j}\right\}_{j=1}^{k}$ are geometrically dual and together generate $H_{1}(\Lambda ; \mathbb{Z})$.

Given such a collection, let $p_{j}$ denote the point of intersection between $m_{j}$ and $l_{j}$ and choose a set of $\operatorname{arcs}\left\{\tau_{j}\right\}_{j=1}^{k}$ in $\Lambda$ intersecting only at a common initial point $* \Lambda$ so that each $\tau_{j}$ intersects the collection of simple closed curves only at its terminal point $p_{j}$; if $\partial \Lambda \neq \varnothing$ choose $*_{\Lambda} \in \partial \Lambda$. Using $\Upsilon=\bigcup_{j=1}^{k} \tau_{j}$ as a "base tree", the curves of $\left\{\left(m_{j}, l_{j}\right)\right\}_{j=1}^{k}$ may be viewed as elements of $\pi_{1}\left(\Lambda, *_{\Lambda}\right)$. In the case where $\partial \Lambda \neq \varnothing$ we may - after relabeling, reordering, and choosing appropriate orientations on the simple closed curves and on $\partial \Lambda$-assume that $\partial \Lambda=\prod_{j=1}^{k} m_{j}^{-1} l_{j}^{-1} m_{j} l_{j}$ in $\pi_{1}(\Lambda, * \Lambda)$.

Remark 2 Since $\Lambda$ is not presumed to bound or be embedded in a 3-manifold, common distinctions between longitude and meridian (or neither) are nonexistent here; a given curve could play either role, depending upon the setup. Nevertheless, the informal use of this terminology will be convenient for discussing certain curves and collections of curves. 
Suppose now that $\Lambda$, with zero or one boundary components and a complete set $\left\{\left(m_{j}, l_{j}\right)\right\}_{j=1}^{k}$ of meridian-longitude pairs, is embedded in an $n$-manifold $N^{n}(n \geq 5)$ and that each $m_{j}$ is homotopically trivial in $N^{n}$. Then we may surger $\Lambda$ to a 2 -sphere or 2-disk in the following manner:

- For convenience, choose a collection $\left\{\bar{m}_{j}\right\}_{j=1}^{k}$ of simple closed curves in $\Lambda$ where each $\bar{m}_{j}$ is parallel to $m_{j}$ and disjoint from $\Upsilon$. Do this so that $\left\{\bar{m}_{j}\right\}_{j=1}^{k}$ is geometrically dual to $\left\{l_{j}\right\}_{j=1}^{k}$.

- Let $\left\{D_{j}\right\}_{j=1}^{k}$ be a collection of pairwise disjoint 2-disks embedded in $N^{n}$ with $\Lambda \cap D_{j}=\partial D_{j}=\bar{m}_{j}$.

- For each $j$, let $A_{j}$ be a small annular neighborhood of $\bar{m}_{j}$ in $\Lambda$ with boundary curves $\bar{m}_{j}^{-}$and $\bar{m}_{j}^{+}$.

- For each $j$, let $D_{j}^{-}$and $D_{j}^{+}$be disks parallel to $D_{j}$ having $\bar{m}_{j}^{-}$and $\bar{m}_{j}^{+}$as boundaries.

- Let $\Lambda^{*}$ be the 2-sphere or 2-disk obtained by removing the interiors of the $A_{j}$ from $\Lambda$ and sewing in $D_{j}^{-}$and $D_{j}^{+}$.

If $\Lambda$ has a preferred orientation, there is a corresponding orientation of $\Lambda^{*}$ where the two agree on $\Lambda-\bigcup A_{j}$. Under that orientation of $\Lambda^{*}$ disk pairs $D_{j}^{ \pm}$inherit opposite orientations when compared by projecting onto $D_{j}$. Suppose $Q^{n-2}$ is a closed oriented submanifold of $N^{n}$ intersecting $\Lambda$ transversely in finitely many points. By applying a small isotopy if necessary, we may assume none of those intersection points is contained in $\bigcup A_{j}$. Adjust the $D_{j}$ (rel boundary) so they also intersect $Q^{n-2}$ transversely. Corresponding to each $p \in D_{j} \cap Q^{n-2}$ there are points $p^{-} \in D_{j}^{-} \cap Q^{n-2}$ and $p^{+} \in D_{j}^{+} \cap Q^{n-2}$. Thus the intersection $\Lambda^{*} \cap Q^{n-2}$ consists of the points of $\Lambda \cap Q^{n-2}$ together with one pair of points $\left\{p^{-}, p^{+}\right\}$for each point $p$ of a $D_{j} \cap Q^{n-2}$. If $N^{n}$ is oriented, it is clear that $\varepsilon_{\mathbb{Z}}\left(p^{-}\right)=-\varepsilon_{\mathbb{Z}}\left(p^{+}\right)$for each of those pairs, so when $\Lambda$ is closed, we have $\varepsilon_{\mathbb{Z}}\left(\Lambda^{*}, Q^{n-2}\right)=\varepsilon_{\mathbb{Z}}\left(\Lambda, Q^{n-2}\right)$.

For $\varepsilon_{\mathbb{Z} \pi_{1}\left(N^{n}, *\right)}\left(\Lambda^{*}, Q^{n-2}\right)$ the situation is more complicated. In order to compare contributions of points $p^{-}$and $p^{+}$, assume the necessary setup discussed in the previous subsection: base points $*, *_{Q}, *_{\Lambda^{*}}=*_{\Lambda}$ and corresponding base paths $\mu_{Q}$ and $\lambda_{\Lambda^{*}}$, and a local orientation at $*$; assume also that $Q^{n-2}$ is simply connected. Determination of $\varepsilon_{\mathbb{Z} \pi_{1}\left(N^{n}, *\right)}\left(p^{-}\right)$and $\varepsilon_{\mathbb{Z} \pi_{1}\left(N^{n}, *\right)}\left(p^{+}\right)$require paths $\rho^{-}$and $\rho^{+}$in $\Lambda^{*}$ from $* \Lambda^{*}$ to $p^{-}$and $p^{+}$. Let $\rho^{-}$be the path in $\Lambda^{*}$ that follows $\tau_{j} \subseteq \Upsilon$ to the longitude $l_{j}$, travels along the unique arc $l_{j}^{-} \subseteq l_{j}$ that arrives at $D_{j}^{-}$without leaving $\Lambda^{*}$, and then travels through $D_{j}^{-}$to $p^{-}$. Choose $\rho^{+}$similarly, noting that $l_{j}^{+}$goes the opposite way around $l_{j}$. Lastly, choose paths $\sigma^{-}$and $\sigma^{+}$in $Q^{n-2}$ from $* Q$ to $p^{-}$ and $p_{+}$; these can be chosen identical except in a small neighborhood of $p$. If we write 
$\varepsilon \mathbb{Z}_{\pi_{1}}(N, *)\left(p^{-}\right)=\operatorname{sgn}\left(p^{-}\right) g$ and $\varepsilon_{\mathbb{Z} \pi_{1}(N, *)}\left(p^{+}\right)=\operatorname{sgn}\left(p^{+}\right) h$ then $g$ is represented by $\lambda_{\Lambda^{*}} \cdot \rho^{-} \cdot\left(\sigma^{-}\right)^{-1} \cdot \mu_{Q}^{-1}$ and $h$ by $\lambda_{\Lambda^{*}} \cdot \rho^{+} \cdot\left(\sigma^{+}\right)^{-1} \cdot \mu_{Q}^{-1}$. It is easy to check that $h=l_{j} g$ in $\pi_{1}(N, *)$ and that $\operatorname{sgn}\left(p^{-}\right)=-\omega_{1}\left(l_{j}\right) \operatorname{sgn}\left(p^{+}\right)$. See Figure 1 .

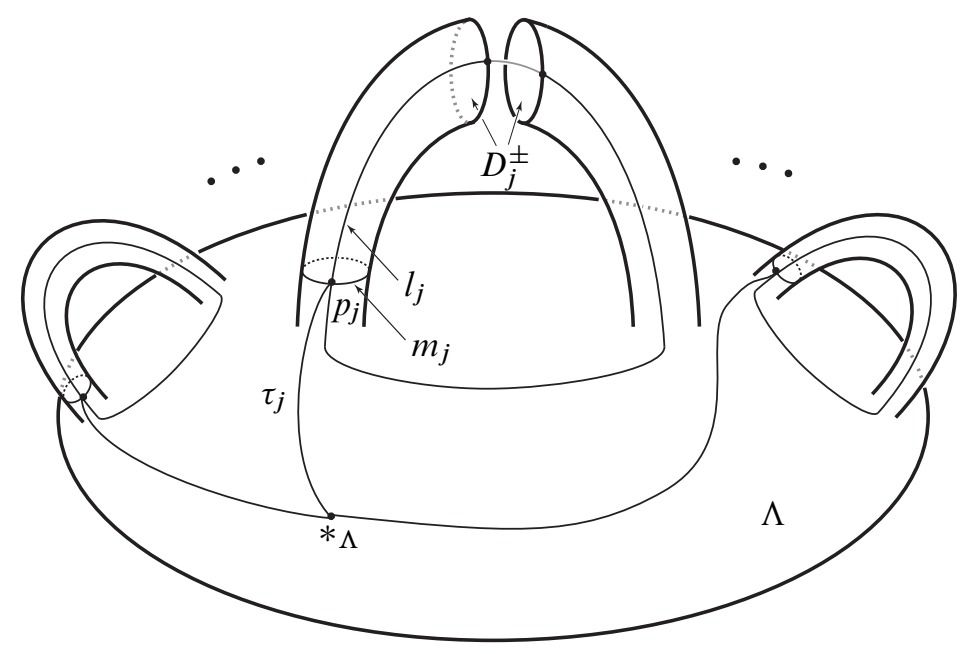

Figure 1: Surgering a surface

So, together this pair of points contributes $\pm\left(1-\omega_{1}\left(l_{j}\right) l_{j}\right) g$ to $\varepsilon_{\mathbb{Z} \pi_{1}\left(N^{n}, *\right)}\left(\Lambda^{*}, Q^{n-2}\right)$. For later reference, we record the following lemma which follows immediately from the above observations.

Lemma 2.1 Let $\left\{\left(m_{j}, l_{j}\right)\right\}_{j=1}^{k}$ a complete set of meridian-longitude pairs for a closed oriented surface $\Lambda$ in the interior of an $n$-manifold $N^{n}$ and let $Q^{n-2}$ be a closed simply connected oriented $(n-2)$-manifold also lying in int $N^{n}$ and intersecting $\Lambda$ transversely. Assume that each of the meridional curves $m_{j}$ contracts in $N^{n}$ and let $\Lambda^{*}$ be a 2 -sphere obtained by surgering $\Lambda$ along a collection of parallel curves; do this in such a way that $\Lambda^{*}$ and $Q^{n-2}$ intersect transversely. Choose base points $*$, $*_{\Lambda}=*_{\Lambda}$, and $*_{Q}$, base paths $\lambda_{\Lambda}$ and $\mu_{Q}$ from $*$ to $*_{\Lambda}$ and $* Q$, respectively and a local orientation of $N^{n}$ at $*$. Then:

(1) If each longitudinal curve $l_{i}$ also contracts in $N^{n}$, then $\varepsilon_{\mathbb{Z} \pi_{1}\left(N^{n}, *\right)}\left(\Lambda, Q^{n-2}\right)$ is well-defined and equal to $\varepsilon \mathbb{Z}_{\pi_{1}}\left(N^{n}, *\right)\left(\Lambda^{*}, Q^{n-2}\right)$.

(2) If $L$ is a normal subgroup of $\pi_{1}\left(N^{n}, *\right)$ with $\omega_{1}(L) \equiv 1$ and each $l_{i}$ represents an element of $L$, then $\varepsilon_{\mathbb{Z}}\left[\pi_{1}\left(N^{n}, *\right) / L\right]\left(\Lambda, Q^{n-2}\right)$ is well-defined and equal to $\varepsilon_{\mathbb{Z}\left[\pi_{1}\left(N^{n}, *\right) / L\right]}\left(\Lambda^{*}, Q^{n-2}\right)$. 


\subsection{Perfect groups and nearly perfect subgroups}

The commutator subgroup of a group $G$, denoted $[G, G]$, is the subgroup generated by all commutator elements $[m, l]=m^{-1} l^{-1} m l$ for $l, m \in G$. It is standard knowledge that $[G, G]$ is a normal and that $G /[G, G]$ is abelian; in fact, $[G, G]$ is the smallest subgroup of $G$ with abelian quotient. If $G=[G, G]$, or equivalently $G /[G, G]$ is trivial, we say $G$ is perfect. In this paper, we are interested in topological implications of these concepts. If $G=\pi_{1}(X, x)$ and $\alpha=\prod_{j=1}^{k}\left[m_{j}, l_{j}\right] \in[G, G]$ then there exists a (mapped in) compact orientable surface $\Lambda_{\alpha}$ with boundary corresponding to $\alpha$ and a base tree for which a complete set of meridian-longitude pairs has the form $\left\{\left(m_{j}, l_{j}\right)\right\}_{j=1}^{k}$. If $\alpha \in K$, where $K$ is a perfect subgroup of $G$, we may arrange that all of the $m_{j}$ and $l_{j}$ are elements of $K$; this is a key property of perfect subgroups of $\pi_{1}(X, x)$.

Next we generalize the notion of "perfectness" for subgroups of $G$. Suppose $K \leq L \leq G$, where $K$ and $L$ are normal in $G$. Then $[K, L]$ is the subgroup of $G$ generated by all elements of the form $[m, l]$ where $m \in K$ and $l \in L$. It is easy to see that $[K, L]=[L, K]$ and $[K, L] \leq K$. We say that $K$ is strongly $L$-perfect if $K \leq[K, L]$. Clearly, $K$ is perfect if and only if it is strongly $K$-perfect; more generally, the smaller the subgroup $L$ containing $K$, the closer a strongly $L$-perfect group is to being perfect. When $G=\pi_{1}(X, x)$, for each element $\alpha$ of a strongly $L$-perfect group $K$, there exists a (mapped in) compact orientable surface $\Lambda_{\alpha}$ with boundary corresponding to $\alpha$ and a base tree for which a complete set of meridian-longitude pairs has the form $\left\{\left(m_{j}, l_{j}\right)\right\}_{j=1}^{k}$, where each $m_{j} \in K$ and $l_{j} \in L$.

Remark 3 We have reserved the term " $L$-perfect" (as compared to "strongly $L-$ perfect") for the case $K \leq[L, L]$, a weaker condition that is developed in [4] but is not used here.

\subsection{Basic handle theory}

Let $N^{n}$ be an $n$-manifold, $B$ a component of $\partial N^{n}$ and $J$ a subset of $B$ homeomorphic to $S^{k-1} \times D^{n-k}$. The act of attaching a $k$-handle (or a handle of index $k$ ) to $N^{n}$ along $J$ is the creation of an adjunction space $N^{n} \cup_{f} D^{n}$, where $D^{n}$ is viewed as $D^{k} \times D^{n-k}$ and $f: S^{k-1} \times D^{n-k} \rightarrow J$ is a homeomorphism. We denote the adjunction space by $N^{n} \cup h^{k}$; here $h^{k}$ denotes the image of $D^{n}$ under the quotient map $q: N^{n} \sqcup D^{n} \rightarrow$ $N^{n} \cup_{f} D^{n}$. We call $J$ the attaching tube of $h^{k}$ and $\alpha^{k-1}=q\left(S^{k-1} \times\{0\}\right)$ the attaching sphere. We call $e^{k}=q\left(D^{k} \times\{0\}\right)$ the core and $q\left(\{0\} \times D^{n-k}\right)$ the cocore of $h^{k}$; the boundary of the cocore, $\beta^{n-k-1}=q\left(\{0\} \times S^{n-k-1}\right)$, is the belt sphere and $q\left(D^{k} \times S^{n-k-1}\right)$ is the belt tube of $h^{k}$. We refer to the boundary component of $N^{n} \cup h^{k}$ consisting of $B-J$ and the belt tube of $h^{k}$ informally as the right-hand 
boundary component. The homeomorphism $f: S^{k-1} \times D^{n-k} \rightarrow J$ is called the framing of $h^{k}$; it affects the homeomorphism type of $N^{n} \cup h^{k}$. More explicitly, if $f_{1}, f_{2}: S^{k-1} \times D^{n-k} \rightarrow J$ is a pair of framings, then the resulting manifolds $N^{n} \cup h_{1}^{k}$ and $N^{n} \cup h_{2}^{k}$ need not be homeomorphic.

The pair $\left(N^{n} \cup h^{k}, N^{n}\right)$ is homotopy equivalent to $\left(N^{n} \cup e^{k}, N^{n}\right)$ in the obvious manner. Thus, if $W^{n}=N^{n} \cup h_{1} \cup \cdots \cup h_{r}$ is obtained from $N^{n}$ by successive attachment of handles of nondecreasing index, each to the right-hand boundary of the preceding space, then $\left(W^{n}, N^{n}\right)$ is homotopy equivalent to a relative $\mathrm{CW}$ complex $\left(K, N^{n}\right)$ with one $j$-cell for each $j$-handle. A useful relationship between these spaces is the equivalence of the $\mathbb{Z}$-incidence number $\varepsilon_{\mathbb{Z}}\left(e^{j+1}, e^{j}\right)$ for a pair of cells $e^{j+1}$ and $e^{j}$ of $K$ and the $\mathbb{Z}$-intersection number $\varepsilon \mathbb{Z}\left(\alpha^{j}, \beta^{n-j-1}\right)$ of the attaching sphere and the belt sphere of corresponding $(j+1)$ - and $j$-handles, and similarly for $\mathbb{Z} \pi_{1}$-incidence and intersection numbers. (Both of these observations require a careful setup involving base points, base paths, orientations, etc. and some special care in dealing with those cases where the attaching or belt sphere is not simply connected. The reader is referred to [6] for details.) The upshot of all this is that intersection numbers, employed appropriately, allow one to calculate algebraic data such as $\mathbb{Z}$-homology, $\mathbb{Z} \pi_{1}$-homology, and Whitehead torsion for manifolds constructed through the addition of handles.

\subsection{Unbased $k$-spheres as elements of $\pi_{k}(N, *)$}

Let $\Sigma_{1}$ and $\Sigma_{2}$ be embedded oriented $k$-spheres $(k \geq 2)$ in a connected manifold $N$, $* \in N, *_{1} \in \Sigma_{1}$, and $*_{2} \in \Sigma_{2}$. Let $S^{k}$ be the standard $k$-sphere with the canonical base point $\mathbf{e}_{1}=(1,0, \ldots, 0)$. In order to view $\Sigma_{1}$ as an element of $\pi_{k}(N, *)$, choose a path $\lambda$ from $*$ to $*_{1}$. Now define a map of $\left(S^{k}, \mathbf{e}_{1}\right)$ into $(N, *)$ as follows. Let $D^{k} \subseteq S^{k}$ be a small $k$-disk centered at $\mathbf{e}_{1}$. Send $\mathbf{e}_{1}$ to $*$ and the radial lines of $D^{k}$ emanating from $\mathbf{e}_{1}$ each onto $\lambda$; then send $S^{k}-D^{k}$ homeomorphically onto $\Sigma_{1}-\left\{*_{1}\right\}$ in an orientation preserving manner. We denote the corresponding element of a $\pi_{k}(N, *)$ by $\left[\lambda \Sigma_{1}\right]$. Stated differently, $\left[\lambda \Sigma_{1}\right]$ is the image of $\left[\Sigma_{1}\right] \in \pi_{k}(N, * 1)$ under the change of base points isomorphism induced by $\lambda$. (See [5, Section 4.1].)

Remark 4 If $*_{1}=*$, then $\lambda$ is a loop and we represent $\left[\lambda \Sigma_{1}\right]$ by $\lambda\left[\Sigma_{1}\right]$, the image of $\left[\Sigma_{1}\right]$ when acted upon by $\lambda$ under well-known action of $\pi_{1}(N, *)$ on $\pi_{k}(N, *)$. Since $\pi_{k}(N, *)$ is abelian, this action may be extended in the obvious way to an action of $Z\left[\pi_{1}(N, *)\right]$ on $\pi_{k}(N, *)$. Again see [5, Section 4.1].

Returning to the original setup, if $\lambda^{\prime}$ is another path from $*$ to $*_{1}$, then $\left[\lambda \Sigma_{1}\right]$ and $\left[\lambda^{\prime} \Sigma_{1}\right]$ need not be equal; it is easy to see that $\left[\lambda^{\prime} \Sigma_{1}\right]=\left(\lambda^{\prime} \cdot \lambda^{-1}\right)\left[\lambda \Sigma_{1}\right]$. 
Now suppose $\xi$ is a path from a point $*^{\prime} \in \Sigma_{1}$ to $*_{2}$. By a strategy similar to the above, we may obtain a map of $S^{k}$ into $N$ sending a slightly shrunken lower hemisphere onto $\Sigma_{1}$, a slightly shrunken upper hemisphere onto $\Sigma_{2}$ (both in orientation preserving manners), and taking a product neighborhood of $S^{k-1}$ onto $\xi$. When the codimension is sufficiently high we may obtain an embedded version of the above, denoted $\Sigma_{1} \#_{\xi} \Sigma_{2}$, it consists of punctured copies of $\Sigma_{1}$ and $\Sigma_{2}$ joined by a "tube" following $\xi$. In either case, we express the corresponding element of $\pi_{k}\left(N, *_{1}\right)$ by $\left[\Sigma_{1} \#_{\xi} \Sigma_{2}\right]$. Returning to our preferred base point we have $\left[\lambda\left(\Sigma_{1} \#_{\xi} \Sigma_{2}\right)\right] \in \pi_{k}(N, *)$, an element that may be expressed as an algebraic sum as follows: choose a path $\sigma$ in $\Sigma_{1}$ from $*_{1}$ to $*^{\prime}$, then $\left[\lambda\left(\Sigma_{1} \#_{\xi} \Sigma_{2}\right)\right]=\left[\lambda \Sigma_{1}\right]+\left[(\lambda \cdot \sigma \cdot \xi) \Sigma_{2}\right]$.

\section{Spherical alteration of a handle}

In this section we give a precise formulation of spherical alteration and prove the corresponding technical lemma. Although the technique can be applied to handles of various indices (usually with some restrictions on codimension), all of our current applications involve alterations of 2-handles. For that reason, we restrict attention to 2-handles and invite the reader to consider possible applications of higher index alterations.

Let $R$ be an $n$-manifold of dimension at least $6, B$ a codimension 0 submanifold of $\partial R, S \approx B \times[0,1]$ a collar neighborhood of $B$ in $R$, and $h^{2}$ a 2-handle attached to the interior boundary component $B_{1}$ of $S$ and lying in $\overline{R-S}$. Let $T=S \cup h^{2}$, $B_{2}=\partial T-B$, and $e^{2}$ the core of $h^{2}$. In addition, let $\Sigma^{2}$ be an oriented 2-sphere embedded in the interior of $R-T$ and $\xi$ an arc in $R$ from a point $p \in e^{2}$ to $q \in \Sigma^{2}$, intersecting $e^{2} \cup \Sigma^{2}$ at no other points. See Figure 2 .

The spherical alteration of $h^{2}$ over $\Sigma^{2}$ along $\xi$ is another 2-handle in $R$ with the same attaching tube as $h^{2}$, but with a core $e^{2} \#_{\xi} \Sigma^{2}$ (the connected sum of $e^{2}$ with $\Sigma^{2}$ along a tube contained in a regular neighborhood of $\xi$ ). We will denote this new 2-handle by $h^{2}\left(\xi, \Sigma^{2}\right)$. An orientation on $e^{2}$ (induced by a preferred characteristic map for $h^{2}$ ) and on $\Sigma^{2}$ are necessary to define $e^{2} \#_{\xi} \Sigma^{2}$; the connecting tube must be chosen to respect these orientations. More precisely, let $E \subseteq E^{\prime} \subseteq e^{2}$ and $F \subseteq \Sigma^{2}$ be small 2-disks centered at $p$ and $q$, and $Z_{\xi}$ an embedded copy of $S^{1} \times[0,1]$ contained in a regular neighborhood of $\xi$ such that $Z_{\xi} \cap e^{2}=\partial E$ and $Z_{\xi} \cap \Sigma^{2}=\partial F$ (at opposite ends of $Z_{\xi}$ ). Then

$$
e^{2} \#_{\xi} \Sigma^{2}=\left(e^{2}-\stackrel{\circ}{E}\right) \cup Z_{\xi} \cup\left(\Sigma^{2}-\stackrel{\circ}{F}\right)
$$




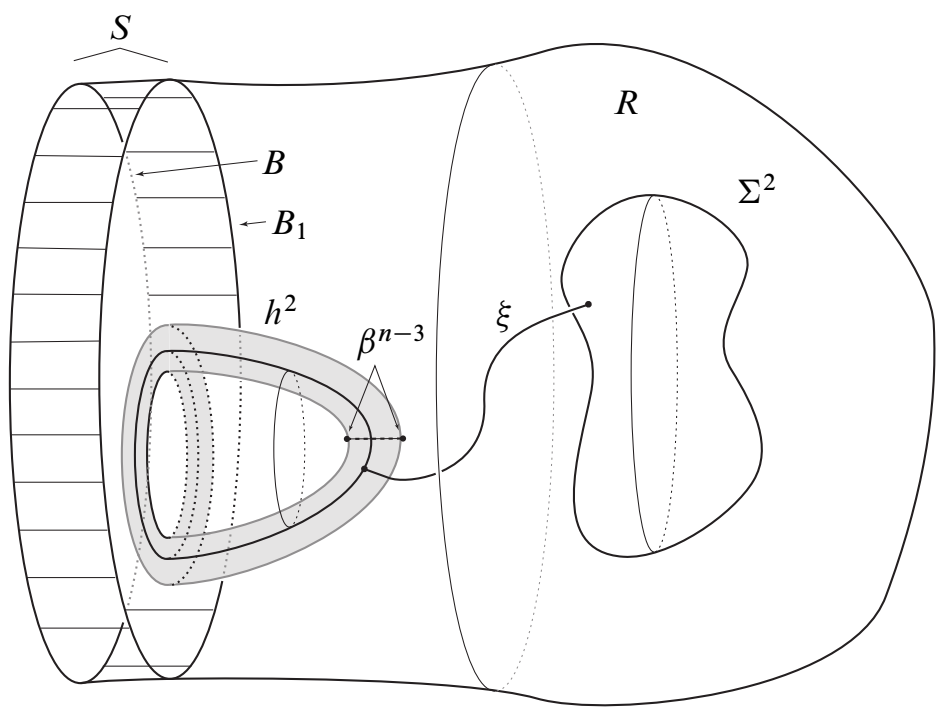

Figure 2: Setup for a spherical alteration

If the orientation on $e^{2}-\stackrel{\circ}{E}$ translated along $Z_{\xi}$ does not match the orientation on $\Sigma^{2}-\stackrel{\circ}{F}$, use the extra codimension to rechoose $Z_{\xi}$ with a twist so that the orientations match.

Use a parameterization of $h^{2}$ as $D^{2} \times D^{n-2}$ to identify $P, \stackrel{\circ}{P} \subseteq h^{2}$ corresponding to $E^{\prime} \times D^{n-2}$ and $\stackrel{\circ}{E}^{\prime} \times D^{n-2}$, respectively; we will refer to $P$ as the alteration region in $h^{2}$. Let $\widehat{h}=h^{2}-\stackrel{\circ}{P}$ and choose a relative regular neighborhood $N$ of the 2-disk

$$
\left(E^{\prime}-\stackrel{\circ}{E}\right) \cup Z_{\xi} \cup\left(\Sigma^{2}-\stackrel{\circ}{F}\right) \subset \overline{R-(S \cup \widehat{h})}
$$

so that $N$ intersects the boundary precisely in $P-\stackrel{\circ}{P} \approx S^{1} \times D^{n-2}$. Define $h^{2}\left(\xi, \Sigma^{2}\right)$ to be $\widehat{h} \cup N$, which is a regular neighborhood of $e^{2} \#_{\xi} \Sigma^{2}$ in $\overline{R-S}$, and thus a 2-handle. Clearly $h^{2}\left(\xi, \Sigma^{2}\right)$ has the same attaching tube as $h^{2}$ (possibly with different framing). We also identify a common belt sphere for $h^{2}$ and $h^{2}\left(\xi, \Sigma^{2}\right)$ lying just outside the alteration region: let $z \in D^{2}$ correspond to a point of $e^{2}$ lying just outside $E^{\prime}$ and let $\beta^{n-3}$ be the $(n-3)$-sphere corresponding to $\partial\left(z \times D^{n-2}\right)$. Finally, let $B_{2}^{\prime}$ denote the right-hand boundary of $T^{\prime}=S \cup h^{2}\left(\xi, \Sigma^{2}\right)$. See Figure 3.

Now assume that, in addition to the above, there is a $2-$ sphere $\Gamma^{2}$ lying in $B_{2}$ and transverse to $\beta^{n-3}$. If $\Gamma^{2}$ and $\beta^{n-3}$ intersect in an essential way, then $\Gamma^{2}$ will not lie in $B_{2}^{\prime}$. Instead, each "sheet" of $\Gamma^{2}$ that cuts through $\beta^{n-3}$ leaves $B_{2}^{\prime}$ at the alteration region. We wish to define an alteration of $\Gamma^{2}$ to a 2 -sphere that lies in $B_{2}^{\prime}$ and intersects $\beta^{n-3}$ in the same way that $\Gamma^{2}$ does. Let $\Gamma^{2} \cap \beta^{n-3}=\left\{p_{1}, \ldots, p_{k}\right\}$. Using the product structure of the belt tube, we may arrange (via an ambient isotopy) that 


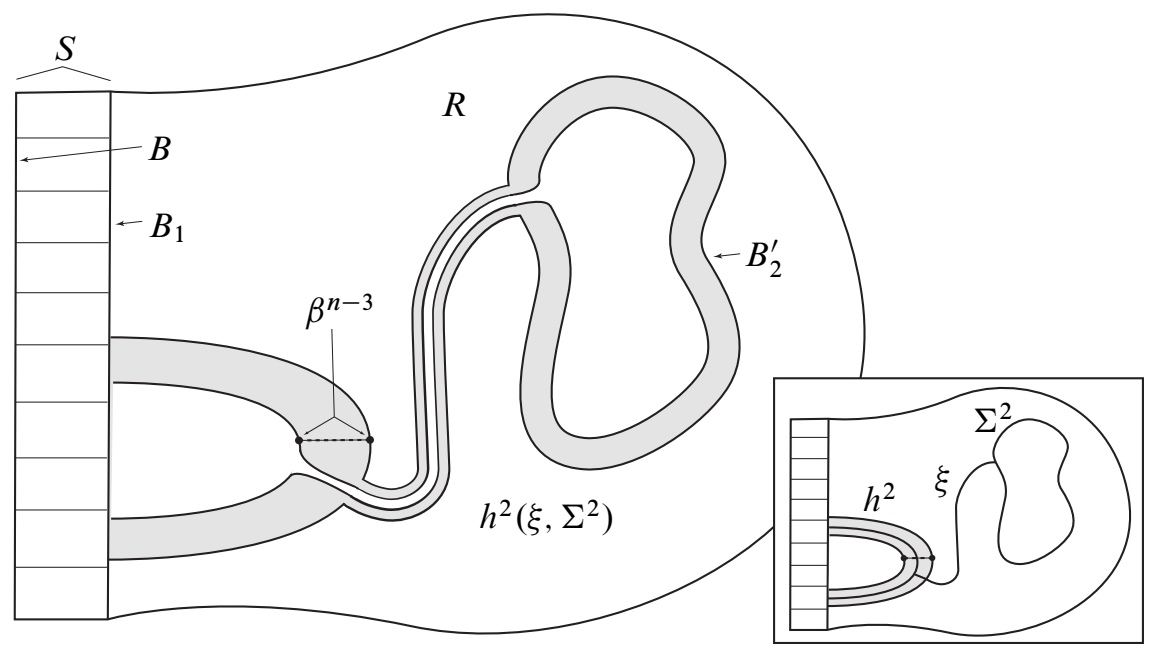

Figure 3: Schematic of a spherical alteration

$\Gamma^{2} \cap h^{2}=\left\{D_{1}, \ldots, D_{k}\right\}$, where the $D_{i}$ are 2-disks in the belt tube parallel to $e^{2}$. Remove from each $D_{i}$ the interior of the subdisk $D_{i}^{\prime}=D_{i} \cap P$, and replace it with an oriented disk $D_{i}^{\prime \prime}$ which has the same boundary (and induces the same orientation on that boundary), but lies in the boundary of the regular neighborhood $N$. Note that $D_{i}^{\prime \prime}$ will be parallel in $N$ to the core of $N$, with the two having matching orientations if $\operatorname{sgn}\left(p_{i}\right)=1$ and opposite orientations when $\operatorname{sgn}\left(p_{i}\right)=-1$. (Orientations are compared by retracting $N$ onto its core.) By general position, we may assume the $D_{i}^{\prime \prime}$ are disjoint; thus we have a new 2-sphere $\Gamma^{2}\left(\xi, \Sigma^{2}\right)$, called the corresponding spherical alteration of $\Gamma^{2}$ over $\Sigma^{2}$ along $\xi$.

Our main lemma equates $\varepsilon_{\mathbb{Z}\left[\pi_{1}\left(B_{2}, *\right)\right]}\left(\Gamma^{2}, \beta^{n-3}\right)$ with $\varepsilon_{\mathbb{Z}\left[\pi_{1}\left(B_{2}^{\prime}, *\right)\right]}\left(\Gamma^{2}\left(\xi, \Sigma^{2}\right), \beta^{n-3}\right)$ and compares $\Gamma^{2}$ and $\Gamma^{2}\left(\xi, \Sigma^{2}\right)$ when viewed as elements of $\pi_{2}(R, *)$. Making our assertions precise requires preliminary work. First, choose $*$ to lie in a portion of $B_{1}$ away from $h^{2}$; then $*$ lies in both $B_{2}$ and $B_{2}^{\prime}$. Although $T$ need not be homeomorphic to $T^{\prime}$ (attaching tubes of the 2-handles are the same, but framings may differ), the fundamental groups are canonically isomorphic: both are obtained by taking the quotient of $\pi_{1}\left(B_{1}, *\right)$ by the normal closure of the common attaching circle for the 2-handles. Since $B_{2} \hookrightarrow T$ and $B_{2}^{\prime} \hookrightarrow T^{\prime}$ induce $\pi_{1}$-isomorphisms, there is a canonical isomorphism $\phi: \pi_{1}\left(B_{2}, *\right) \rightarrow \pi_{1}\left(B_{2}^{\prime}, *\right)$ that associates each loop in $B_{2}$ missing the alteration region (a collection that generates the entire fundamental group) to the identical loop in $B_{2}^{\prime}$. In a casual sense, $\phi$ may be viewed as an identity map. Let $\widehat{\phi}: \mathbb{Z}\left[\pi_{1}\left(B_{2}, *\right)\right] \rightarrow \mathbb{Z}\left[\pi_{1}\left(B_{2}^{\prime}, *\right)\right]$ be the corresponding "identity-like" ring isomorphism. 
In order to calculate $\varepsilon \mathbb{Z}\left[\pi_{1}\left(B_{2}, *\right)\right]\left(\Gamma^{2}, \beta^{n-3}\right)$ we select some initial data. Assume that $\left\{p_{1}, \ldots, p_{k}\right\}$ is nonempty and choose $p_{1}$ as base point for both $\Gamma^{2}$ and $\beta^{n-3}$. Choose a local orientation for $B_{2}$ at $*$ and a path $\lambda$ in $B_{2}$ from $*$ to $p_{1}$ to serve as base path for both $\Gamma^{2}$ and $\beta^{n-3}$. The remaining data needed for $\varepsilon_{\mathbb{Z}\left[\pi_{1}\left(B_{2}, *\right)\right]}\left(\Gamma^{2}, \beta^{n-3}\right)$ is a collection of paths $\rho_{i}$ in $\Gamma^{2}$ and $\sigma_{i}$ in $\beta^{n-3}$ and from $p_{1}$ to $p_{i}$ for each $i=2, \ldots, k$. ( $\rho_{1}$ and $\sigma_{1}$ can be constant paths). When calculating $\varepsilon \mathbb{Z}\left[\pi_{1}\left(B_{2}^{\prime}, *\right)\right]\left(\Gamma^{2}\left(\xi, \Sigma^{2}\right), \beta^{n-3}\right)$ notice that $\Gamma^{2}\left(\xi, \Sigma^{2}\right) \cap \beta^{n-3}=\left\{p_{1}, \ldots, p_{k}\right\}$ and that the local orientation at $*$ and each of the points and paths just selected can be chosen to lie simultaneously in $B_{2}$ and $B_{2}^{\prime}$; one simply avoids the alteration region. By using the same initial data for both, it is immediate that $\varepsilon_{\mathbb{Z}}\left[\pi_{1}\left(B_{2}, *\right)\right]\left(\Gamma^{2}, \beta^{n-3}\right)$ is the same as $\varepsilon_{\mathbb{Z}\left[\pi_{1}\left(B_{2}^{\prime}, *\right)\right]}\left(\Gamma^{2}\left(\xi, \Sigma^{2}\right), \beta^{n-3}\right)$; more precisely, $\widehat{\phi}$ takes the former to the latter.

Two more items are needed in preparation for the statement of our main lemma. First, for each $i=1, \ldots, k$, choose a path $\xi_{i}$ from $p_{i}$ to the point $q \in \Sigma^{2}$ which travels the short distance through the belt tube from $p_{i}$ to the alteration region, then runs parallel to $\xi$ and ends at $q$. It is then clear that each $\xi_{i}$ is homotopic in $R$ (rel endpoints) to $\sigma_{i}^{-1} \cdot \xi_{1}$. Finally, the inclusion $j: B_{2} \hookrightarrow R$ induces a group homomorphism

$$
j_{\#}: \pi_{1}\left(B_{2}, *\right) \rightarrow \pi_{1}(R, *)
$$

and a corresponding ring homomorphism

$$
\widehat{j}_{\#}: \mathbb{Z}\left[\pi_{1}\left(B_{2}, *\right)\right] \rightarrow \mathbb{Z}\left[\pi_{1}(R, *)\right]
$$

that plays a role in the following.

Lemma 3.1 (Spherical alteration) Given the spherical alteration of $h^{2}$ over $\Sigma^{2}$ along $\xi$ described above, the corresponding alteration of $\Gamma^{2}$, and all of the base point, path, and homomorphism data selected in the previous three paragraphs, the following is true:

(1) $\varepsilon_{\mathbb{Z}\left[\pi_{1}\left(B_{2}, *\right)\right]}\left(\Gamma^{2}, \beta^{n-3}\right)$ is taken to $\varepsilon_{\mathbb{Z}\left[\pi_{1}\left(B_{2}^{\prime}, *\right)\right]}\left(\Gamma^{2}\left(\xi, \Sigma^{2}\right), \beta^{n-3}\right)$ by

$$
\widehat{\phi}: \mathbb{Z}\left[\pi_{1}\left(B_{2}, *\right)\right] \rightarrow \mathbb{Z}\left[\pi_{1}\left(B_{2}^{\prime}, *\right)\right] .
$$

(2) As elements of $\pi_{2}(R, *),\left[\lambda \Gamma^{2}\left(\xi, \Sigma^{2}\right)\right]$ is equal to

$$
\left[\lambda \Gamma^{2}\right]+\widehat{j}_{\#}\left(\varepsilon_{\mathbb{Z}\left[\pi_{1}\left(B_{2}, *\right)\right]}\left(\Gamma^{2}, \beta^{n-3}\right)\right)\left[\left(\lambda \cdot \xi_{1}\right) \Sigma^{2}\right] .
$$

Proof Item (1) was covered in the lead-up to this lemma.

As for item (2), it is easy to see that the embedding which takes $S^{2}$ onto $\Gamma^{2}\left(\xi, \Sigma^{2}\right)$ is homotopic in $R$ to the map indicated by

$$
\Gamma^{2} \#_{\xi_{1}}\left(\operatorname{sgn}\left(p_{1}\right) \Sigma^{2}\right) \#_{\xi_{2}}\left(\operatorname{sgn}\left(p_{2}\right) \Sigma^{2}\right) \#_{\xi_{3}} \cdots \#_{\xi_{k}}\left(\operatorname{sgn}\left(p_{k}\right)\right) \Sigma^{2},
$$


where a minus sign indicates a reversed orientation. By repeatedly applying the observations made in Section 2.5, we see that

$$
\begin{aligned}
{\left[\lambda \left(\Gamma^{2} \#_{\xi_{1}}\right.\right.} & \left.\left.\left(\operatorname{sgn}\left(p_{1}\right) \Sigma^{2}\right) \#_{\xi_{2}}\left(\operatorname{sgn}\left(p_{2}\right) \Sigma^{2}\right) \#_{\xi_{3}} \cdots \#_{\xi_{k}}\left(\operatorname{sgn}\left(p_{k}\right)\right) \Sigma^{2}\right)\right] \\
& =\left[\lambda \Gamma^{2}\right]+\left[\left(\lambda \cdot \xi_{1}\right) \operatorname{sgn}\left(p_{1}\right) \Sigma^{2}\right]+\sum_{i=2}^{k} \operatorname{sgn}\left(p_{i}\right)\left[\left(\lambda \cdot \rho_{i} \cdot \xi_{i}\right) \Sigma^{2}\right] \\
& =\left[\lambda \Gamma^{2}\right]+\operatorname{sgn}\left(p_{1}\right)\left[\left(\lambda \cdot \xi_{1}\right) \Sigma^{2}\right]+\sum_{i=2}^{k} \operatorname{sgn}\left(p_{i}\right)\left[\left(\lambda \cdot \rho_{i} \cdot\left(\sigma_{i}^{-1} \cdot \xi_{1}\right)\right) \Sigma^{2}\right] \\
& =\left[\lambda \Gamma^{2}\right]+\operatorname{sgn}\left(p_{1}\right)\left[\left(\lambda \cdot \xi_{1}\right) \Sigma^{2}\right]+\sum_{i=2}^{k} \operatorname{sgn}\left(p_{i}\right)\left[\left(\lambda \cdot \rho_{i} \cdot \sigma_{i}^{-1} \cdot(\lambda-1 \cdot \lambda) \cdot \xi_{1}\right) \Sigma^{2}\right] \\
& =\left[\lambda \Gamma^{2}\right]+\operatorname{sgn}\left(p_{1}\right)\left[\left(\lambda \cdot \xi_{1}\right) \Sigma^{2}\right]+\sum_{i=2}^{k} \operatorname{sgn}\left(p_{i}\right)\left(\lambda \cdot \rho_{i} \cdot \sigma_{i}^{-1} \cdot \lambda^{-1}\right)\left[\left(\lambda \cdot \xi_{1}\right) \Sigma^{2}\right] \\
& =\left[\lambda \Gamma^{2}\right]+\operatorname{sgn}\left(p_{1}\right)\left[\left(\lambda \cdot \xi_{1}\right) \Sigma^{2}\right]+\sum_{i=2}^{k} \operatorname{sgn}\left(p_{i}\right) g_{i}\left[\left(\lambda \cdot \xi_{1}\right) \Sigma^{2}\right] \\
& =\left[\lambda \Gamma^{2}\right]+\left(\operatorname{sgn}\left(p_{1}\right)+\sum_{i=2}^{k} \operatorname{sgn}\left(p_{i}\right) g_{i}\right)\left[\left(\lambda \cdot \xi_{1}\right) \Sigma^{2}\right],
\end{aligned}
$$

where $g_{i}=\lambda \cdot \rho_{i} \cdot \sigma_{i}^{-1} \cdot \lambda^{-1}$ is precisely the loop used in defining $\varepsilon_{\mathbb{Z}\left[\pi_{1}\left(B_{2}, *\right)\right]}\left(p_{i}\right)$ for $i=2, \ldots, k$. By our choice of base paths, the loop corresponding to $p_{1}$ is null-homotopic, so $\varepsilon_{\mathbb{Z}\left[\pi_{1}\left(B_{2}, *\right)\right]}\left(p_{1}\right)=\operatorname{sgn}\left(p_{1}\right)$. Thus

$$
\left(\operatorname{sgn}\left(p_{1}\right)+\sum_{i=2}^{k} \operatorname{sgn}\left(p_{i}\right) g_{i}\right)=\varepsilon_{\mathbb{Z}\left[\pi_{1}\left(B_{2}, *\right)\right]}\left(\Gamma^{2}, \beta^{n-3}\right) .
$$

The inclusion of each $g_{i}$ into $(R, *)$ yields $j_{\#}\left(g_{i}\right)$, thereby converting

$$
\operatorname{sgn}\left(p_{1}\right)+\sum_{i=2}^{k} \operatorname{sgn}\left(p_{i}\right) g_{i} \quad \text { to } \widehat{j}_{\#}\left(\operatorname{sgn}\left(p_{1}\right)+\sum_{i=2}^{k} \operatorname{sgn}\left(p_{i}\right) g_{i}\right) .
$$

So the lemma is proved.

\section{The embedded manifold plus construction}

In this section we employ the method of spherical alteration described above to obtain a constructive proof of Theorem 4.2. An indirect proof, relying on the $s$-cobordism 
theorem, was given in [4]. An advantage to the current approach is that it may be modified to obtain more general results; that is the content of the last two sections of this paper. A side benefit of the constructive proof is that it yields a proof of the classical manifold plus construction which avoids many subtleties related to framings and bundle theory.

We begin by reviewing a proof of the classical manifold plus construction. Suppose $(W, A, B)$ is a compact cobordism between closed manifolds and $A \hookrightarrow W$ is a homotopy equivalence, in which case we call $(W, A, B)$ a one-sided $h$-cobordism. An application of Poincaré duality in the universal cover (see [3, Theorem 2.5]) shows that $\pi_{1}(B) \rightarrow \pi_{1}(W)$ is surjective with perfect kernel. The manifold plus construction provides a converse to that observation.

Theorem 4.1 (Manifold plus construction) Let $B$ be a closed $(n-1)$-manifold $(n \geq 6)$ and $\theta: \pi_{1}(B, *) \rightarrow H$ a surjective homomorphism onto a finitely presented group such that $\operatorname{ker} \theta$ is perfect. Then there exists a compact one-sided $h$-cobordism $(W, A, B)$ such that $\operatorname{ker}\left(\pi_{1}(B, *) \rightarrow \pi_{1}(W, *)\right)=\operatorname{ker} \theta$. In fact, it may be arranged that $A \hookrightarrow W$ is a simple homotopy equivalence, in which case $W$ unique up to homeomorphism rel $B$.

A one-sided $h$-cobordism $(W, A, B)$ for which the homotopy equivalence $A \hookrightarrow W$ is simple will be called a plus cobordism. To avoid repetition, we adopt the convention that whenever a one-sided (or plus) cobordism is discussed, it will be the first of the two boundary components listed, ie the middle term in the triple, which includes into $W$ as a (simple) homotopy equivalence.

A classical proof of Theorem 4.1 Step I (Attaching 2-handles to kill $\operatorname{ker} \theta$ ) Associate $B$ with $B \times\{0\} \subseteq S=B \times\left[\overline{0,1]}\right.$ and let $B_{1}=B \times\{1\}$. By a standard group theoretic argument, $\operatorname{ker} \theta$ is the normal closure of a finite set of elements of $\pi_{1}(B, *)$; identify a corresponding collection of nicely embedded oriented loops $\left\{\alpha_{i}\right\}_{i=1}^{r}$ in $B_{1}$. Since we are dealing with a normal closure, we need not be concerned with base points, so we may assume the loops are pairwise disjoint. Since all elements of $\operatorname{ker} \theta$ are homologically trivial, each $\alpha_{i}$ has a regular neighborhood in $B_{1}$ homeomorphic to $S^{1} \times D^{n-2}$. Identify a pairwise disjoint collection $\left\{J_{i}\right\}_{i=1}^{r}$ of such neighborhoods and use them as attaching tubes for a set $\left\{h_{i}^{2}\right\}_{i=1}^{r}$ of 2 -handles. For the moment, we do not concern ourselves with the framings of those 2 -handles. The resulting $n$-manifold $T$ has fundamental group isomorphic to $H$. By inverting these handles, $T$ may be obtained by attaching a collection of $(n-2)$-handles to a collar neighborhood of $B_{2}=\partial T-B$, a process that does not change fundamental group, so $B_{2} \hookrightarrow T$ induces 
a $\pi_{1}$-isomorphism. Note, however, that $H_{n-2}\left(T, B_{2} ; \mathbb{Z}\right) \cong \mathbb{Z}^{r}$, so we do not have a one-sided $h$-cobordism.

Step II (Attaching complementary 3-handles) Here we will attach a collection of 3-handles that are complementary to the above 2-handles (in an appropriately strong sense) so that the end result is the desired cobordism $(W, A, B)$. Along the way, we may need to rechoose the framings of the 2-handles attached in Step I. Let $\left\{\beta_{i}^{n-3}\right\}_{i=1}^{r}$ be the collection of belt spheres of $\left\{h_{i}^{2}\right\}_{i=1}^{r}$. Our initial goal is to identify a pairwise disjoint collection $\left\{\Gamma_{i}^{2}\right\}_{i=1}^{r}$ of 2 -spheres in $B_{2}$ that is algebraically dual over $\mathbb{Z}\left[\pi_{1}\left(B_{2}, *\right)\right]$ to $\left\{\beta_{i}^{n-3}\right\}_{i=1}^{r}$. In order to utilize the $\left\{\Gamma_{i}^{2}\right\}_{i=1}^{r}$ as attaching spheres for a collection of 3-handles $\left\{h_{i}^{3}\right\}_{i=1}^{r}$, we must also ensure that each has a regular neighborhood homeomorphic to $S^{2} \times D^{n-3}$ in $B_{2}$. Once that is accomplished, our task is essentially complete; we will let $W=B \times[0,1] \cup\left\{h_{i}^{2}\right\}_{i=1}^{r} \cup\left\{h_{i}^{3}\right\}_{i=1}^{r}$ and $A=\partial W-B$. Inverting that handle decomposition, we may view $W$ as a collar on $A$ together with a collection of $(n-3)$-handles $\left\{h_{i}^{n-3}\right\}_{i=1}^{r}$ (the duals of the 3-handles) with belt spheres $\left\{\Gamma_{i}^{2}\right\}_{i=1}^{r}$ and a collection of $(n-2)$-handles $\left\{h_{i}^{n-2}\right\}_{i=1}^{r}$ (the duals of the 2-handles) with attaching spheres $\left\{\beta_{i}^{n-3}\right\}_{i=1}^{r}$. Then $A \hookrightarrow W$ induces a $\pi_{1}$-isomorphism with each fundamental group isomorphic to $H$; moreover, the intersection data (as discussed in Section 2.4) tells us that the corresponding cellular $\mathbb{Z}\left[\pi_{1}(A)\right]$-complex for the pair $(W, A)$ is of the form

$$
0 \rightarrow \widetilde{C}_{n-2} \stackrel{\partial_{n-2}}{\longrightarrow} \widetilde{C}_{n-3} \rightarrow 0 \rightarrow \cdots \rightarrow 0,
$$

where each of $\widetilde{C}_{n-2}$ and $\widetilde{C}_{n-3}$ is isomorphic to a free $\mathbb{Z}\left[\pi_{1}(A)\right]$-module on $r$ generators and, with respect to the obvious preferred bases, the boundary operator $\partial_{n-2}$ can be represented by a diagonal matrix with diagonal entries all being \pm 1 . It follows that $A \hookrightarrow W$ is a simple homotopy equivalence.

We now turn to the construction of $\left\{\Gamma_{i}^{2}\right\}_{i=1}^{r}$. This is the heart of the matter; it is where the beauty of the plus construction lies. Since each $\alpha_{i}$ represents an element of the perfect group $\operatorname{ker} \theta$ it may be expressed as $\prod_{j=1}^{k_{i}}\left[m_{j}^{i}, l_{j}^{i}\right]$, where $\left\{\left(m_{j}^{i}, l_{j}^{i}\right)\right\}_{j=1}^{k_{i}}$ is a complete set of meridian-longitude pairs for a compact orientable surface $\Lambda_{i} \subseteq B_{1}$ and each $m_{j}^{i}$ and $l_{j}^{i}$ also lies in $\operatorname{ker} \theta$. Using general position and the radial structure of the attaching tubes $J_{i}$, we can adjust these surfaces so that each has boundary $\alpha_{i}^{\prime}$ which lies in $\partial J_{i}$ and is parallel to $\alpha_{i}$. In addition we may assume that the $\Lambda_{i}$ are properly embedded in $B_{1}-\bigcup_{i=1}^{r}$ int $J_{i}$ and pairwise disjoint. Complete each $\Lambda_{i}$ to a closed surface $\widehat{\Lambda}_{i} \subseteq B_{2}$ by adding a 2 -disk $\widehat{D}_{i}$ lying in the belt tube of $h_{i}^{2}$ and parallel to its core. It is here that we must pay attention to framings. Since $\Lambda_{i}$ is orientable and deformation retracts onto a bouquet of circles, where each of those circles corresponds to an $m_{j}^{i}$ or an $l_{j}^{i}$, all of which have trivial normal bundles, standard 
bundle theory can be used to verify that $\Lambda_{i}$ has a product relative regular neighborhood in $B_{1}-\bigcup_{i=1}^{r}$ int $J_{i}$. If necessary, we now rechoose the framing used to attach $h_{i}^{2}$ so that the corresponding trivial normal bundle for $\widehat{D}_{i}$ matches up with that of $\Lambda_{i}$ to give $\widehat{\Lambda}_{i}$ a product regular neighborhood in $B_{2}$; indeed, this is precisely the matter which determines the framings that must be used for attaching the 2-handles. (One may argue that this should have been discussed before attaching the 2-handles; however, it seems instructive to discover the issue in context.)

Notice that each surface $\widehat{\Lambda}_{i}$ intersects the belt sphere $\beta_{i}^{n-3}$ transversely in exactly one point and that it intersects no other belt spheres. Thus, $\left\{\widehat{\Lambda}_{i}\right\}$ is a collection of geometric duals for $\left\{\beta_{i}^{n-3}\right\}$, and since the fundamental group of each $\widehat{\Lambda}_{i}$ includes trivially into $B_{2}$, this may be expressed in terms of $\mathbb{Z}\left[\pi_{1}\left(B_{2}\right)\right]$-intersection numbers. After choosing all necessary base points, base paths, and orientations the geometric intersection properties imply that $\varepsilon \mathbb{Z}\left[\pi_{1}\left(B_{2}\right)\right]\left(\widehat{\Lambda}_{i}, \beta_{j}^{n-3}\right)=0$ whenever $i \neq j$ and each $\varepsilon \mathbb{Z}\left[\pi_{1}\left(B_{2}\right)\right]\left(\widehat{\Lambda}_{i}, \beta_{i}^{n-3}\right)= \pm g_{i}$ for some $g_{i} \in \mathbb{Z}\left[\pi_{1}\left(B_{2}\right)\right]$. We may arrange that each of the latter intersection numbers is \pm 1 by rechoosing some of the base paths.

Unfortunately, the $\widehat{\Lambda}_{i}$ will usually have genus $>0$, and thus be unusable for attaching 3 -handles. We remedy that problem by surgering the surfaces to 2 -spheres in the manner outlined in Section 2.2. Since each $m_{j}^{i}$ contracts in $B_{2}$ we may surger $\widehat{\Lambda}_{i}$ to a 2 -sphere $\Gamma_{i}$ in $B_{2}$ using disks bounded by the various meridional curves. (In the notation of Section 2.2, $\Gamma_{i}=\widehat{\Lambda}_{i}^{*}$.) By Lemma 2.1(1) this operation preserves $\mathbb{Z}\left[\pi_{1}\left(B_{2}\right)\right]$-intersection numbers, so $\varepsilon_{\mathbb{Z}\left[\pi_{1}\left(B_{2}\right)\right]}\left(\beta_{i}^{n-3}, \Gamma_{j}\right)= \pm \delta_{i j}$ for all $1 \leq i, j \leq r$. Another application of standard bundle theory ensures that the $\Gamma_{i}$ inherit trivial normal bundles from the $\widehat{\Lambda}_{i}$, so they may be used as attaching spheres for the 3 -handles $\left\{h_{i}^{3}\right\}_{i=1}^{r}$, thereby supplying the final ingredient of the manifold plus construction.

The uniqueness part of this theorem follows from a clever application of the $s-$ cobordism theorem. Since it is not of primary importance to this paper, we refer the reader to [1, page 197] for a proof.

We are now ready for the embedded version of the manifold plus construction. Much of the strategy and notation employed above is recycled into the proof, the main ideas are the same. Some issues become more complex due to our desire to embed the construction in an ambient manifold; as a pleasant surprise, other issues become easier for the same reason.

Theorem 4.2 (Embedded manifold plus construction) Let $R$ be an $n$-manifold $(n \geq 6)$ containing a closed $(n-1)$-manifold $B$ in its boundary and suppose

$$
\operatorname{ker}\left(i_{*}: \pi_{1}(B, *) \longrightarrow \pi_{1}(R, *)\right)
$$


contains a perfect group $G$ that is the normal closure in $\pi_{1}(R, *)$ of a finite set of elements. Then there exists an embedding of a plus cobordism $(W, A, B)$ into $R$ which is the identity on $B$ and for which $\operatorname{ker}\left(\pi_{1}(B) \rightarrow \pi_{1}(W)\right)=G$.

Proof Step I (Finding embedded 2-handles that kill ker $i_{\#}$ ) Let $S \approx B \times[0,1]$ be a collar neighborhood of $B$ in $R$ and let $B_{1}$ denote the interior boundary component of $S$. Choose a pairwise disjoint collection of properly embedded 2-disks $\left\{D_{1}, \ldots, D_{r}\right\}$ in $\overline{R-S}$ whose boundaries in $B_{1}$ represent a finite normal generating set for $G$. By taking regular neighborhoods, thicken the $D_{i}$ to a pairwise disjoint collection of 2-handles $\left\{h_{i}^{2}\right\}_{i=1}^{r}$. Let $T=S \cup h_{1}^{2} \cup \cdots \cup h_{r}^{2}$ and $B_{2}=\partial T-B$; for later use, let $J_{i}$ denote the attaching tube for $h_{i}^{2}$. Then $\pi_{1}\left(B_{2}\right) \cong \pi_{1}(T) \cong \pi_{1}(B) / G$ and $\operatorname{ker}\left(\pi_{1}(B) \rightarrow \pi_{1}(T)\right)=G$. For the remainder of the proof, all work will be done within a regular neighborhood $R^{\prime}$ of $T$ in $R$. Since $R^{\prime}$ is just $T$ with a collar added along $B_{2}, B_{2} \hookrightarrow \overline{R^{\prime}-T}$ induces a $\pi_{1}$-isomorphism, a fact that will be utilized only in the special argument needed for the $n=6$ case.

Step II (Altering the embedded 2-handles so that complementary embedded 3-handles $\overline{\text { exist) }}$ We would like to find a pairwise disjoint collection of 3-handles $\left\{h_{i}^{3}\right\}_{i=1}^{r}$ embedded in $\overline{R^{\prime}-T}$ with attaching 2 -spheres algebraically dual over $\mathbb{Z}\left[\pi_{1}\left(B_{2}\right)\right]$ to the collection $\left\{\beta_{i}^{n-3}\right\}_{i=1}^{r}$ of belt spheres of $\left\{h_{i}^{2}\right\}_{i=1}^{r}$. Adding those handles to $T$ (and following the argument used in the previous theorem) would give us the desired $W \subseteq R^{\prime}$.

Toward that end goal, we construct a collection $\left\{\Gamma_{i}\right\}_{i=1}^{r}$ of 2 -spheres in $B_{2}$ which are algebraic duals for the collection $\left\{\beta_{i}^{n-3}\right\}_{i=1}^{r}$ in precisely the same manner employed in Step II of the previous theorem. (But unlike that proof, we need not concern ourselves with framings of the 2-handles or regular neighborhoods of the 2-spheres.)

Under ideal circumstances, the $\left\{\Gamma_{i}\right\}_{i=1}^{r}$ would contract in $\overline{R^{\prime}-T}$ allowing us to obtain a pairwise disjoint collection of properly embedded 3-disks in $\overline{R^{\prime}-T}$ with the $\Gamma_{i}$ as boundaries. Thickening those disks to 3 -handles would complete the construction of $W$.

The main strategy of this proof can now be described: by utilizing a carefully selected sequence of spherical modifications of the $\left\{h_{i}^{2}\right\}_{i=1}^{r}$ we arrive at a new collection of embedded 2-handles so that the correspondingly altered versions of the $\left\{\Gamma_{i}\right\}_{i=1}^{r}$ satisfy the desired contractibility condition.

Step $\mathrm{II}_{1}$ (Spherical alteration of $h_{1}^{2}$ ) Locate a parallel copy of $\Gamma_{1}$ lying in the interior of the space $\overline{R^{\prime}-T}$. (Push $\Gamma_{1}$ along collar lines.) Reverse the orientation on that copy and denote it by $\Delta_{1}$. Choose a base point $p_{1}$ for $\Gamma_{1}$ lying in the belt tube of $h_{1}^{2}$ but 
missing the belt sphere $\beta_{1}^{n-3}$, and let $\xi_{1}$ be the track of $p_{1}$ in $\overline{R^{\prime}-T}$ under the push. Extend $\xi_{1}$ slightly to an arc $\xi_{1}^{\prime}$ which connects the core of $h_{1}^{2}$ to $\Delta_{1}$, and perform a spherical alteration of $h_{1}^{2}$ over $\Delta_{1}$ along $\xi_{1}^{\prime}$ to obtain $h_{1}^{2}\left(\xi_{1}^{\prime}, \Delta_{1}\right)$. Then perform the corresponding spherical alterations on each $2-$ sphere in the collection $\left\{\Gamma_{i}\right\}_{i=1}^{r}$ (Note: Although the algebraic intersection number of $\Gamma_{i}$ with $\beta_{1}^{n-3}$ is 0 when $i \geq 2$, the two need not be disjoint; so the alterations must be done in order to obtain a collection that lies in the right-hand boundary $B_{2}^{(1)}$ of $T^{(1)}=S \cup h_{1}^{2}\left(\xi_{1}^{\prime}, \Delta_{1}\right) \cup h_{2}^{2} \cup \cdots \cup h_{r}^{2}$.) Now make the following observations:

(a 1$)$ The collection $\left\{\Gamma_{i}^{2}\left(\xi_{1}^{\prime}, \Delta_{1}\right)\right\}_{i=1}^{r}$ of altered 2-spheres is algebraically dual to the set $\left\{\beta_{i}^{n-3}\right\}_{i=1}^{r}$ of belt spheres of the new collection of 2 -handles

$$
\left\{h_{1}^{2}\left(\xi_{1}^{\prime}, \Delta_{1}\right), h_{2}^{2}, \ldots, h_{r}^{2}\right\}
$$

in $B_{2}^{(1)}$. (Recall that the belt sphere $\beta_{1}^{n-3}$ of $h_{1}^{2}$ is also the belt sphere for $h_{1}^{2}\left(\xi_{1}^{\prime}, \Delta_{1}\right)$.) We need only check that

$$
\varepsilon_{\mathbb{Z}\left[\pi_{1}\left(B_{2}^{(1)}, *\right)\right]}\left(\beta_{i}^{n-3}, \Gamma_{j}^{2}\left(\xi_{1}^{\prime}, \Delta_{1}\right)\right)=\varepsilon_{\mathbb{Z}\left[\pi_{1}\left(B_{2}, *\right)\right]}\left(\beta_{i}^{n-3}, \Gamma_{j}\right)
$$

for all $1 \leq i, j \leq r$. But this is clear, since $\Gamma_{i}^{2}\left(\xi_{1}^{\prime}, \Delta_{1}\right)$ and $\Gamma_{i}$ are identical over path connected subsets which contain all points of intersection with elements of $\left\{\beta_{i}^{n-3}\right\}_{i=1}^{r}$. This means that all paths and loops utilized in determining the two intersection numbers can be chosen to be identical; such loops represent the "same" elements of $\pi_{1}\left(B_{2}^{(1)}\right)$ as they do in $\pi_{1}\left(B_{2}\right)$.

(b $\left.{ }_{1}\right) \quad \Gamma_{1}^{2}\left(\xi_{1}^{\prime}, \Delta_{1}\right)$ contracts in $R^{\prime}$. To see this, let $\lambda_{1}$ be a path from the base point $p_{0}$ of $R^{\prime}$ to $p_{1}$, then by Lemma 3.1, as elements of $\pi_{2}\left(R^{\prime}, p_{0}\right)$,

$$
\begin{aligned}
{\left[\lambda_{1} \Gamma_{1}^{2}\left(\xi_{1}^{\prime}, \Delta_{1}\right)\right] } & =\left[\lambda_{1} \Gamma_{1}^{2}\right]+\left[\left(\lambda_{1} \cdot \xi_{1}\right)\left(\Delta_{1}\right)\right] \\
& =\left[\lambda_{1} \Gamma_{1}^{2}\right]+\left[\lambda_{1}\left(-\Gamma_{1}^{2}\right)\right] \\
& =\left[\lambda_{1} \Gamma_{1}^{2}\right]-\left[\lambda_{1}\left(\Gamma_{1}^{2}\right)\right]=0 .
\end{aligned}
$$

So $\Gamma_{1}^{2}\left(\xi_{1}^{\prime}, \Delta_{1}\right)$ contracts in $R^{\prime}$.

$\underline{\text { Step } \mathrm{II}_{2}}\left(\right.$ Spherical alteration of $\left.h_{2}^{2}\right)$ Now begin with the collection of 2-handles

$$
\left\{h_{1}^{2}\left(\xi_{1}^{\prime}, \Delta_{1}\right), h_{2}^{2}, \ldots, h_{r}^{2}\right\}
$$

attached to $S$ in $R^{\prime}$ and the collection $\left\{\Gamma_{i}^{2}\left(\xi_{1}^{\prime}, \Delta_{1}\right)\right\}_{i=1}^{r}$ of algebraic duals to their belt spheres $\left\{\beta_{i}^{n-3}\right\}_{i=1}^{r}$ in $B_{2}^{(1)}$. Obtain a $2-$ sphere $\Delta_{2}$ by pushing the element $\Gamma_{2}\left(\xi_{1}^{\prime}, \Delta_{1}\right)$ into the interior of the space $\overline{R^{\prime}-T^{(1)}}$ and then reversing its orientation. Let $p_{2}$ be an appropriately chosen base point for $\Gamma_{2}\left(\xi_{1}^{\prime}, \Delta_{1}\right)$ and $\xi_{2}$ be the track of $p_{2}$ in $\overline{R^{\prime}-T^{(1)}}$ 
under the push. Extend $\xi_{2}$ slightly to an arc $\xi_{2}^{\prime}$ which connects the core of $h_{2}^{2}$ to $\Delta_{2}$, and perform the spherical alteration of $h_{2}^{2}$ over $\Delta_{2}$ along $\xi_{2}^{\prime}$ to obtain $h_{2}^{2}\left(\xi_{2}^{\prime}, \Delta_{2}\right)$. Then perform the corresponding spherical alterations on each 2 -sphere in the collection $\left\{\Gamma_{i}^{2}\left(\xi_{1}^{\prime}, \Delta_{1}\right)\right\}_{i=1}^{r}$. To save on notation, we denote the twice altered version of $\Gamma_{i}^{2}$ by $\Gamma_{i}^{2}\left(\bigsqcup_{j=1}^{2} \xi_{j}^{\prime}, \Delta_{j}\right)$. Let $T^{(2)}=S \cup h_{1}^{2}\left(\xi_{1}^{\prime}, \Delta_{1}\right) \cup h_{2}^{2}\left(\xi_{2}^{\prime}, \Delta_{2}\right) \cup h_{3}^{2} \ldots h_{r}^{2}$. Using the same arguments as above, we now have:

(a 2$)$ The collection $\left\{\Gamma_{i}^{2}\left(\bigsqcup_{j=1}^{2} \xi_{j}^{\prime}, \Delta_{j}\right)\right\}_{i=1}^{r}$ of twice altered 2-spheres is algebraically dual to the set $\left\{\beta_{i}^{n-3}\right\}_{i=1}^{r}$ of belt spheres of the new collection of 2-handles

$$
\left\{h_{1}^{2}\left(\xi_{1}^{\prime}, \Delta_{1}\right), h_{2}^{2}\left(\xi_{2}^{\prime}, \Delta_{2}\right), h_{3}^{2}, \ldots, h_{r}^{2}\right\}
$$

in $B_{2}^{(2)}$.

$\left(b_{2}\right)$ Both

$$
\Gamma_{1}^{2}\left(\bigsqcup_{j=1}^{2} \xi_{j}^{\prime}, \Delta_{j}\right) \text { and } \Gamma_{2}^{2}\left(\bigsqcup_{j=1}^{2} \xi_{j}^{\prime}, \Delta_{j}\right)
$$

contract in $R^{\prime}$. (Contractibility of $\Gamma_{1}^{2}\left(\bigsqcup_{j=1}^{2} \xi_{j}^{\prime}, \Delta_{j}\right.$ ) follows from Lemma 3.1 and the fact that $\varepsilon_{\mathbb{Z} \pi_{1}\left(B_{2}^{(1)}\right)}\left(\beta_{2}^{n-3}, \Gamma_{1}^{2}\left(\xi_{1}^{\prime}, \Delta_{1}\right)\right)=0$.)

Continue the above process until each of $h_{1}^{2}, \ldots, h_{r}^{2}$ has been altered by a similar process, and let $T^{(r)}$ denote the union of $S$ and these altered 2-handles. At this point we have:

$\left(\mathrm{a}_{r}\right)$ The collection

$$
\left\{\Gamma_{i}^{2}\left(\bigsqcup_{j=1}^{r} \xi_{j}^{\prime}, \Delta_{j}\right)\right\}_{i=1}^{r}
$$

of $r$-times altered 2-spheres is algebraically dual to the set $\left\{\beta_{i}^{n-3}\right\}_{i=1}^{r}$ of belt spheres of the handles $\left\{h_{i}^{2}\left(\xi_{i}^{\prime}, \Delta_{i}\right)\right\}_{i=1}^{r}$ in $B_{2}^{(r)}$.

$\left(\mathrm{b}_{r}\right) \quad \Gamma_{1}^{2}\left(\bigsqcup_{j=1}^{r} \xi_{j}^{\prime}, \Delta_{j}\right), \ldots, \Gamma_{r}^{2}\left(\bigsqcup_{j=1}^{r} \xi_{j}^{\prime}, \Delta_{j}\right)$ contract in $R^{\prime}$.

Before proceeding to the final stage of our proof, let us simplify the above notation. From now on each of the altered 2-handles $h_{i}^{2}\left(\xi_{i}^{\prime}, \Delta_{i}\right)$ will be denoted by $\dot{h}_{i}^{2}$ and each of the $r$-times altered 2-spheres $\Gamma_{i}^{2}\left(\bigsqcup_{j=1}^{r} \xi_{j}^{\prime}, \Delta_{j}\right)$ by $\dot{\Gamma}_{i}^{2}$. Thus, we have

$$
T^{(r)}=S \cup \dot{h}_{1}^{2} \cup \cdots \cup \dot{h}_{r}^{2} \subseteq R^{\prime}
$$

and a collection $\left\{\dot{\Gamma}_{i}^{2}\right\}_{i=1}^{r}$ of 2 -spheres algebraically dual over $\mathbb{Z}\left[\pi_{1}\left(B_{2}^{(r)}, *\right)\right]$ to the collection $\left\{\beta_{i}^{n-3}\right\}_{i=1}^{r}$ of belt spheres of those 2 -handles in $B_{2}^{(r)}$. In addition, each $\dot{\Gamma}_{i}^{2}$ 
contracts in $R^{\prime}$. By general position, these 2-spheres also contract in $\overline{R^{\prime}-T^{(r)}}$. This is because any contraction of $\dot{\Gamma}_{i}^{2}$ in $R^{\prime}$ can be pushed off the 2-dimensional cores of all of the 2-handles and, thus, entirely out of the interior of $T^{(r)}$. Assume for the moment that the dimension of $R^{\prime}$ is at least 7 , Then we may choose a pairwise disjoint collection $\left\{D_{i}^{3}\right\}_{i=1}^{r}$ of properly embedded 3-disks in $\overline{R^{\prime}-T^{(r)}}$ such that $\partial D_{i}^{3}=\dot{\Gamma}_{i}^{2}$ for each $i=1, \ldots, r$. Take pairwise disjoint relative regular neighborhoods of these 3-disks in $\overline{R^{\prime}-T^{(r)}}$ to obtain 3-handles $\left\{\dot{h}_{i}^{3}\right\}_{i=1}^{r}$ in $\overline{R^{\prime}-T^{(r)}}$ attached to $B_{2}^{(r)}$. Let

$$
W=T^{(r)} \cup\left(\bigcup_{i=1}^{r} \dot{h}_{i}^{3}\right)=S \cup\left(\bigcup_{i=1}^{r} \dot{h}_{i}^{2}\right) \cup\left(\bigcup_{i=1}^{r} \dot{h}_{i}^{3}\right)
$$

and let $A=\partial W-B$. By the same reasoning used in Theorem $4.1,(W, A, B)$ is a plus cobordism.

Step III ( $\pi-\pi$ argument for the $n=6$ case) The only place the above proof runs into

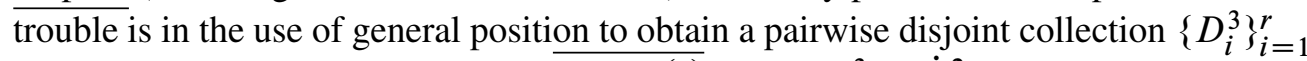
of properly embedded 3-disks in $\overline{R^{\prime}-T^{(r)}}$ with $\partial D_{i}^{3}=\dot{\Gamma}_{i}^{2}$ for each $i$. If $n=6$, we may use general position to obtain a collection of immersed 3-disks $\left\{\widetilde{D}_{i}^{3}\right\}_{i=1}^{r}$, each containing a finite collection of interior transverse self-intersection points, and a finite number of interior points where it transversely intersects another member of the collection. We will employ a well-known strategy (see, for example, the proof of the $\pi-\pi$ Theorem in [9, Chapter 4]) to eliminate all intersection and self-intersection points. Once that is accomplished, the proof may be completed in the previous manner. Let $p \in \widetilde{D}_{i}^{3} \cap \widetilde{D}_{j}^{3}$ for some $i \neq j$. Choose arcs $\alpha$ and $\alpha^{\prime}$ in $\widetilde{D}_{i}^{3}$ and $\widetilde{D}_{j}^{3}$ respectively

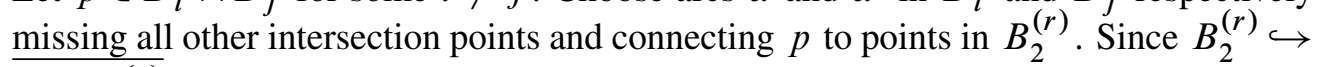
$\overline{R^{\prime}-T^{(r)}}$ induces a $\pi_{1}$-isomorphism (a surjection is sufficient), we may connect the endpoints of $\alpha$ and $\alpha^{\prime}$ by an $\operatorname{arc} \alpha^{\prime \prime}$ in $B_{2}^{(r)}$ such that the loop $\alpha \cup \alpha^{\prime} \cup \alpha^{\prime \prime}$ contracts in $\overline{R^{\prime}-T^{(r)}}$. By general position we may choose an embedded 2-disk $\delta$ bounded by $\alpha \cup \alpha^{\prime} \cup \alpha^{\prime \prime}$ which intersects the collection $\left\{\widetilde{D}_{k}^{3}\right\}_{k=1}^{r} \widetilde{D}^{3}$ only at $\alpha \cup \alpha^{\prime}$, and intersects $B_{2}^{(r)}$ only at $\alpha^{\prime \prime}$. Use $\delta$ to define a proper isotopy of $\widetilde{D}_{i}^{3}$ in $R^{\prime}-T^{(r)}$ which moves points in a small neighborhood of $\alpha$ across $\delta$ to the other side of $\alpha^{\prime}$, thus eliminating the point of intersection $p$. See Figure 4.

Alternatively, perform a "finger move" on $\widetilde{D}_{i}^{3}$ along the arc $\alpha^{\prime \prime}$ to create a new point $p^{\prime}$ of transverse intersection between $\widetilde{D}_{i}^{3}$ and $\widetilde{D}_{j}^{3}$. The disk $\delta$ contains a Whitney disk which allows us to simultaneously remove $p$ and $p^{\prime}$ via an isotopy of $\widetilde{D}_{i}^{3}$ (after finger move) which takes place entirely in a neighborhood of $\delta$. Similar procedures may be used to eliminate a point of self-intersection from a given $\widetilde{D}_{i}^{3}$.

Apply the above procedure to each point of intersection between distinct elements of $\left\{\widetilde{D}_{k}^{3}\right\}_{k=1}^{r}$ and each point of self-intersection of each $\widetilde{D}_{i}^{3}$ to arrive at a pairwise disjoint 

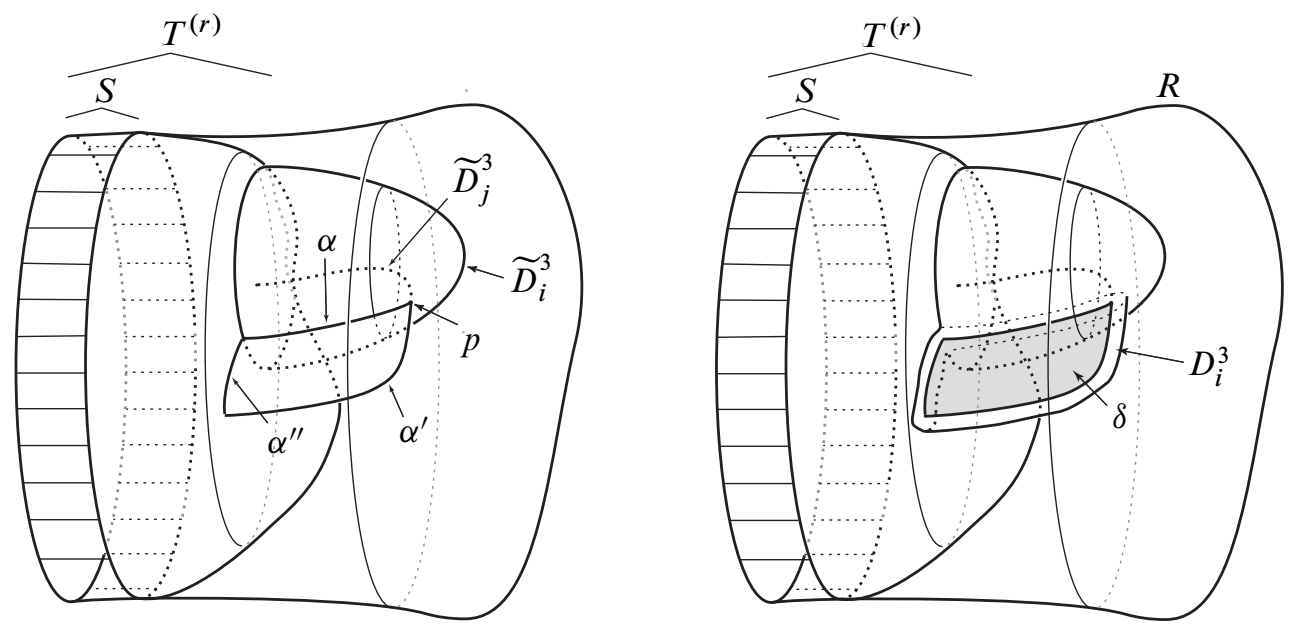

Figure 4: Eliminating a point of intersection using the $\pi-\pi$ procedure

collection of properly embedded 3-disks $\left\{D_{k}^{3}\right\}_{k=1}^{r}$. Note that the boundary of each $D_{k}^{3}$ is isotopic to the original $\dot{\Gamma}_{k}^{2}$ in $B_{2}^{(r)}$. Hence, the $\left\{\partial D_{k}^{3}\right\}_{k=1}^{r}$ is still a collection of algebraic duals for the $\left\{\beta_{k}^{n-3}\right\}_{k=1}^{r}$ in $B_{2}^{(r)}$.

Remark 5 The reader will note that a key issue in the proof of Theorem 4.1 - the existence of product neighborhoods for the 2-spheres along which the 3-handles will be attached - does not appear in the proof of Theorem 4.2. In the latter setting, the 3-handles are realized as regular neighborhoods of embedded 3-disks; as such, product neighborhoods of their boundaries are guaranteed by regular neighborhood theory.

This is the essence of our alternate proof of Theorem 4.1. One first carries out Step I of the classical proof; in particular, construct a manifold $T$ by attaching finitely many 2-handles to $B \times[0,1]$ to kill $\operatorname{ker} \theta$ (and with no attention given to the framings used). Theorem 4.2 applied to the inclusion $i: B \hookrightarrow T$ with $G=\operatorname{ker} i_{*}=\operatorname{ker} \theta$ then assures the existence of the desired plus cobordism lying inside $T$. It strikes us as surprising that the full plus cobordism can be found embedded in the first stage of that construction, even when the first stage is done with the wrong framings.

\section{Generalized manifold plus constructions and their embed- dings}

The techniques employed in the proofs of Theorems 4.1 and 4.2 can be carried out without the full hypothesis of "perfectness" on the subgroups $\operatorname{ker} \theta$ and $G$, provided 
one is satisfied with weaker (but still useful) conclusions. In this section we develop results of that type. Primary motivation for the definitions and results found here is provided by our ongoing study of noncompact manifolds [2].

Our first goal is to formulate appropriate generalizations of "one-sided $h$-cobordism" and "plus cobordism". Let $(X, A)$ be a connected CW pair and $L \unlhd \pi_{1}(A)$. The inclusion $A \hookrightarrow X$ is a $(\bmod L)$-homotopy equivalence if it induces an isomorphism on fundamental groups and is a $\mathbb{Z}\left[\pi_{1}(A) / L\right]$-homology equivalence; if that homology equivalence is simple we call $A \hookrightarrow X$ is a $(\bmod L)$-simple homotopy equivalence.

A compact cobordism $(W, A, B)$ is a $(\bmod L)$-one-sided $h$-cobordism if $B \hookrightarrow W$ induces a surjection of fundamental groups and $A \hookrightarrow W$ is a $(\bmod L)$-homotopy equivalence. A one-sided $(\bmod L)-h$-cobordism for which $A \hookrightarrow W$ is a $(\bmod L)-$ simple homotopy equivalence is called a $(\bmod L)$-plus cobordism.

Remark 6 Standard arguments show that the notions of $(\bmod L)$-homotopy equivalence, $(\bmod L)$-one-sided $h$-cobordism, and $(\bmod L)$-plus cobordism reduce to the classical definitions when $L=\{1\}$. In that case the surjectivity of $\pi_{1}(B) \rightarrow \pi_{1}(W)$ is automatic and need not be included in the definitions (see the second paragraph of Section 4). When $L$ is nontrivial that condition must be included in the definition to obtain a theory that parallels the classical situation. For example, it provides a natural correspondence between $\pi_{1}(A) / L$ and $\pi_{1}(B) / L^{\prime}$ where $L^{\prime}$ is the preimage of $L$; from there it follows (by Poincaré duality) that $B \hookrightarrow W$ is also a $Z\left[\pi_{1}(A) / L\right]-$ homology equivalence.

The following provides an important connection between $(\bmod L)$-one-sided $h$ cobordisms and the material presented in Section 2.3.

Lemma 5.1 Let $(W, A, B)$ is a $(\bmod L)$-one-sided $h$-cobordism and $i_{\#}: \pi_{1}(B) \rightarrow$ $\pi_{1}(W)$ the inclusion induced surjection. Then $\operatorname{ker} i_{\#}$ is strongly $L^{\prime}$-perfect, where $L^{\prime}=i_{\#}^{-1}(L)$.

Proof This follows from Poincare duality and the 5-term exact sequence from the theory of group homology [7;8]. See [2] for a detailed proof.

We are now ready to state and prove generalizations of the two main theorems from the previous section.

Theorem 5.2 (Generalized manifold plus construction) Let $B$ be a closed $(n-1)-$ manifold $(n \geq 6)$ and $\theta: \pi_{1}(B, *) \rightarrow H$ a surjective homomorphism onto a finitely presented group such that $\operatorname{ker} \theta$ is strongly $L^{\prime}$-perfect for some group $L^{\prime}$ where $\operatorname{ker} \theta \unlhd L^{\prime} \unlhd \pi_{1}(B, *)$ and $\omega_{1}\left(L^{\prime}\right)=1$. Then, for $L=L^{\prime} / \operatorname{ker} \theta$, there exists a compact $(\bmod L)$-plus cobordism $(W, A, B)$ such that $\operatorname{ker}\left(\pi_{1}(B, *) \rightarrow \pi_{1}(W, *)\right)=\operatorname{ker} \theta$. 
Theorem 5.3 (Generalized embedded manifold plus construction) Let $R$ be an $n$-manifold $(n \geq 6)$ containing a closed $(n-1)$-manifold $B$ in its boundary and suppose $\pi_{1}(B, *)$ contains a pair of normal subgroups $G \leq L^{\prime}$, each contained in $\operatorname{ker}\left(i_{*}: \pi_{1}(B, *) \longrightarrow \pi_{1}(R, *)\right)$, such that $G$ is strongly $L^{\prime}$-perfect. Suppose also that $G$ is the normal closure in $\pi_{1}(B, *)$ of a finite set of elements. Then, for $L=L^{\prime} / G$, there exists an embedding of a $(\bmod L)$-plus cobordism $(W, A, B)$ into $R$ which is the identity on $B$ and for which $\operatorname{ker}\left(\pi_{1}(B) \rightarrow \pi_{1}(W)\right)=G$.

Proofs of each of these theorems can be obtained by reworking those from the previous section with the new weaker hypotheses, obtaining correspondingly weaker conclusions. We sketch out the details of those changes needed to obtain Theorem 5.2 and leave it to the reader to carry out the analogous changes required to obtain Theorem 5.3.

Sketch of the generalized manifold plus construction Begin by repeating Step I of the proof of Theorem 4.1; in particular, attach a collection $\left\{h_{i}^{2}\right\}_{i=1}^{r}$ of 2-handles to $B_{1}=B \times\{1\} \subseteq S=B \times[0,1]$ to kill a finite set $\left\{\alpha_{i}\right\}_{i=1}^{r}$ of oriented loops which normally generate $\operatorname{ker} \theta$. Let $T=S \cup\left(\bigcup_{i=1}^{r} h_{i}^{2}\right)$. Moving to Step II, strong $L^{\prime}-$ perfectness ensures that each $\alpha_{i}$ bounds a compact oriented surface $\Lambda_{i} \subseteq B_{1}$ which contains a complete set $\left\{\left(m_{j}^{i}, l_{j}^{i}\right)\right\}_{j=1}^{k_{i}}$ of meridian-longitude pairs for which each $m_{j}^{i}$ corresponds to an element of $\operatorname{ker} \theta$ and each $l_{j}^{i}$ to an element of $L^{\prime}$. Using the same general position and bundle-theoretic arguments employed earlier, add a disk to each $\Lambda_{i}$ to obtain a pairwise disjoint collection $\left\{\widehat{\Lambda}_{i}\right\}_{i=1}^{r}$ of closed oriented surfaces in $B_{2}=$ $\partial T-B$ geometrically dual to the collection $\left\{\beta_{i}^{n-3}\right\}_{i=1}^{r}$ of belt spheres of the $\left\{h_{i}^{2}\right\}_{i=1}^{r}$. By rechoosing the framings of the 2-handles if necessary, we may arrange that each $\widehat{\Lambda}_{i}$ has a product neighborhood in $B_{2}$; here we utilize the hypothesis that $\omega\left(L^{\prime}\right)=1$. Since each $\widehat{\Lambda}_{i}$ has a fundamental group that includes into $L \unlhd \pi_{1}\left(B_{2}\right)$, the $\mathbb{Z}\left[\pi_{1}\left(B_{2}\right) / L\right]-$ intersection numbers between elements of $\left\{\widehat{\Lambda}_{i}\right\}_{i=1}^{r}$ and $\left\{\beta_{i}^{n-3}\right\}_{i=1}^{r}$ are well-defined. By making appropriate choices of local orientation and base paths, we may arrange that $\varepsilon \mathbb{Z}\left[\pi_{1}\left(B_{2}\right) / L\right]\left(\widehat{\Lambda}_{i}, \beta_{j}^{n-3}\right)= \pm \delta_{i j}$ for all $1 \leq i, j \leq r$. By applying Lemma 2.1(2), these surfaces may be surgered into a collection of 2-spheres $\left\{\Gamma_{i}\right\}_{i=1}^{r}$ which is algebraically dual over $\mathbb{Z}\left[\pi_{1}\left(B_{2}\right) / L\right]$ to the collection $\left\{\beta_{i}^{n-3}\right\}$. Standard bundle theory again ensures that the $\Gamma_{i}$ inherit trivial normal bundles from the $\widehat{\Lambda}_{i}$. Attach 3 -handles along regular neighborhoods of these 2 -spheres to obtain a cobordism $(W, A, B)$. Then $A \hookrightarrow W$ induces a $\pi_{1}$-isomorphism, with $\pi_{1}(A) \cong \pi_{1}(W) \cong \pi_{1}\left(B_{2}\right)$ and the above intersection data assures that $A \hookrightarrow W$ is a simple $\mathbb{Z}\left[\pi_{1}(A) / L\right]$-equivalence. The surjectivity of $\pi_{1}(B) \rightarrow \pi_{1}(W)$ is clear from the construction.

Sketch of the generalized embedded manifold plus construction For the most part, this proof follows the same outline as the proof of Theorem 4.2 with modifications 
analogous to those found in the above sketch. A few items become more delicate; we focus our attention on those issues.

(1) In the proof of Theorem 4.2 we carried out the entire construction inside a regular neighborhood $R^{\prime}$ of $T$, chosen early in the proof. That was done solely for use in Step III. There it was crucial that $B_{2}^{(r)} \hookrightarrow \overline{R^{\prime}-T^{(r)}}$ induce a $\pi_{1}$-surjection, thereby allowing us to choose an arc $\alpha^{\prime \prime}$ in $B_{2}^{(r)}$ so that the loop $\alpha \cup \alpha^{\prime} \cup \alpha^{\prime \prime}$ contracted in $\overline{R^{\prime}-T^{(r)}}$. In the more general case at hand, it will be impossible to carry out the entire construction in a regular neighborhood of $T$. Instead, we will expand the region where we work to an open set $R^{\prime \prime} \supseteq T$ in which all loops corresponding to elements of $L^{\prime}$ contract and for which $B_{2} \hookrightarrow \overline{R^{\prime \prime}-\text { int } T}$ induces a $\pi_{1}$-surjection. If $L^{\prime}$ is the normal closure in $\pi_{1}(B, *)$ of a finite set of its elements, this is easy: Let $S \approx$ $B \times[0,1]$ be a collar neighborhood of $B$ in $R$ with $B_{1}$ the interior boundary component. Choose a pairwise disjoint collection of properly embedded 2 -disks $\left\{D_{1}, \ldots, D_{r}\right\}$ in $\overline{R-S}$ whose boundaries in $B_{1}$ represent a finite normal generating set for $G$, then supplement that collection with a disjoint collection of pairwise disjoint 2-disks $\left\{D_{r+1}, \ldots, D_{s}\right\}$ in $\overline{R-S}$ whose boundaries, together with those of $\left\{D_{1}, \ldots, D_{r}\right\}$, form a normal generating set for $L^{\prime}$. Then $T$ may be viewed as a regular neighborhood of $S \cup\left(\bigcup_{i=1}^{r} D_{i}\right)$ in $R$ and we may let $R^{\prime \prime}$ be a regular neighborhood of $S \cup\left(\bigcup_{i=1}^{S} D_{i}\right)$ chosen to contain $T$ in its interior.

When $L^{\prime}$ is not normally finitely generated we use a similar, but more delicate construction. Choose an infinite collection of 2-disks $\left\{D_{r+1}, D_{r+2}, \ldots\right\}$ whose boundaries, together with those of $\left\{D_{1}, \ldots, D_{r}\right\}$ generate $L^{\prime}$. These may be chosen inductively so that each $D_{i}$ has a neighborhood $U_{i}$ for which the collection $\left\{U_{i}\right\}_{i=1}^{\infty}$ is pairwise disjoint. We may then thicken each $D_{i}(i>r)$ to a $2-$ handle $h_{i}^{2} \subseteq U_{i}$ and add to a slightly enlarged copy of $T$ the interiors of each of these 2 -handles. This creates an open subset $R^{\prime \prime}$ of $R$ containing $T$ and having the desired properties.

(2) Following the same strategy sketched out in the proof of Theorem 5.2, but utilizing the more delicate item (2) of Lemma 2.1, we obtain a collection of 2 -spheres $\left\{\Gamma_{i}\right\}_{i=1}^{r}$ in $B_{2}$ which is algebraically dual over $\mathbb{Z}\left[\pi_{1}\left(B_{2}\right) / L\right]$ to the collection $\left\{\beta_{i}^{n-3}\right\}_{i=1}^{r}$. (Note that $\omega_{1}\left(L^{\prime}\right) \equiv 1$ since $L^{\prime} \leq \operatorname{ker}\left(i_{*}: \pi_{1}(B, *) \longrightarrow \pi_{1}(R, *)\right)$.) Next we proceed inductively through the spherical alteration process in the same manner as Step II of the proof of Theorem 4.2 so that, at the conclusion, we have a new set of 2 -handles $\left\{\dot{h}_{i}\right\}_{i=1}^{r}$ in $R^{\prime \prime}$ attached to $S$ and a collection $\left\{\dot{\Gamma}_{i}^{2}\right\}_{i=1}^{r}$ of $2-$ spheres algebraically dual over $\mathbb{Z}\left[\pi_{1}\left(B_{2}^{(r)}, *\right) / L\right]$ to the collection $\left\{\beta_{i}^{n-3}\right\}_{i=1}^{r}$ of belt spheres of those 2 -handles in $B_{2}^{(r)}$. In addition, each $\dot{\Gamma}_{i}^{2}$ contracts in $R^{\prime \prime}$. Contractibility of the $2-$ spheres is more delicate in this generalized situation. We use the full strength of Lemma 3.1(2), the key point being that $j_{\#}: \pi_{1}\left(B_{2}, *\right) \rightarrow \pi_{1}\left(R^{\prime \prime}, *\right)$ is precisely the homomorphism that 
kills $L \unlhd \pi_{1}\left(B_{2}, *\right)$. By general position these 2 -spheres also contract in $\overline{R^{\prime \prime}-T^{(r)}}$, so for $n \geq 7$ we may thicken a corresponding collection of pairwise disjoint 3-disks to 3 -handles to complete the construction of $W$.

(3) For $n=6$, Step III of the proof Theorem 4.2 goes through without any changes. It is here, however, where we use the carefully chosen set $R^{\prime \prime}$ in which to carry out the construction.

\section{A more general lemma}

The following technical lemma was specifically designed for use in [2]. It is more general than Theorem 5.3, but no new ideas or techniques are needed. For the reader who has made it this far, the proof is almost immediate.

Lemma 6.1 Let $R^{\prime} \subseteq R$ be a pair of $n$-manifolds $(n \geq 6)$ with a common boundary component $B$, and suppose there is a subgroup $L^{\prime}$ of $\operatorname{ker}\left(\pi_{1}(B) \rightarrow \pi_{1}(R)\right)$ for which $K=\operatorname{ker}\left(\pi_{1}(B) \rightarrow \pi_{1}\left(R^{\prime}\right)\right)$ is strongly $L^{\prime}$-perfect. Suppose further that there is a clean submanifold $T \subseteq R^{\prime}$ consisting of a finite collection $\mathcal{H}^{2}$ of 2-handles in $R^{\prime}$ attached to a collar neighborhood $S$ of $B$ with $T \hookrightarrow R^{\prime}$ inducing a $\pi_{1}$-isomorphism (the 2-handles precisely kill the group $K$ ) and a finite collection $\left\{\Theta_{t}^{2}\right\}$ of pairwise disjoint embedded 2-spheres in $\partial T-B$, each of which contracts in $R^{\prime}$. Then on any subcollection $\left\{h_{j}^{2}\right\}_{j=1}^{k} \subseteq \mathcal{H}^{2}$, one may perform spherical alterations to obtain 2-handles $\left\{\dot{h}_{j}^{2}\right\}_{j=1}^{k}$ in $R^{\prime}$ so that in $\partial \dot{T}-B$ (where $\dot{T}$ is the correspondingly altered version of $T$ ) there is a collection of $2-$ spheres $\left\{\dot{\Gamma}_{j}^{2}\right\}_{j=1}^{k}$ that are algebraically dual over $\mathbb{Z}\left[\pi_{1}(B) / L^{\prime}\right]$ to the belt spheres $\left\{\beta_{j}^{n-3}\right\}_{j=1}^{k}$ common to $\left\{h_{j}^{2}\right\}_{j=1}^{k}$ and $\left\{\dot{h}_{j}^{2}\right\}_{j=1}^{k}$ with the property that each $\dot{\Gamma}_{j}^{2}$ contracts in $R$. Furthermore, each correspondingly altered 2 -sphere $\dot{\Theta}_{t}^{2}$ (now lying in $\partial \dot{T}-B$ ) has the same $\mathbb{Z}\left[\pi_{1}(B) / L^{\prime}\right]$-intersection number with those belt spheres and with any other oriented $(n-3)$-manifold lying in both $\partial T-B$ and $\partial \dot{T}-B$ as did $\Theta_{t}^{2}$. Whereas the 2 -spheres $\left\{\Theta_{t}^{2}\right\}$ each contracted in $R^{\prime}$, the $\left\{\dot{\Theta}_{t}^{2}\right\}$ each contract in $R$.

Remark 7 In the usual way, when $n \geq 7$, the above result together with general position assures the existence of a pairwise disjoint collection of embedded 3-disks in $\overline{R-\dot{T}}$ with boundaries corresponding to the 2 -spheres $\left\{\dot{\Gamma}_{j}^{2}\right\}_{j=1}^{k} \cup\left\{\dot{\Theta}_{t}^{2}\right\}$. Those 3 -disks may be thickened to 3 -handles by taking regular neighborhoods. When $n=6$, the same is true, but the $\pi-\pi$ argument used in the proofs of Theorems 4.2 and 5.3 must again be employed to obtain embedded and pairwise disjoint 3-disks. In that case we should also use the strategy used in the proof of Theorem 5.3 to ensure that we are working within a submanifold $R^{\prime \prime}$ of $R$ which contains $T$ and in which the group $L^{\prime}$ dies and $\pi_{1}(B) \rightarrow \pi_{1}\left(R^{\prime \prime}\right)$ is surjective. 


\section{References}

[1] M H Freedman, F Quinn, Topology of 4-manifolds, Princeton Mathematical Series 39, Princeton Univ. Press (1990) MR1201584

[2] C R Guilbault, F C Tinsley, Manifolds that are inward tame at infinity, In progress

[3] C R Guilbault, F C Tinsley, Manifolds with non-stable fundamental groups at infinity, II, Geom. Topol. 7 (2003) 255-286 MR1988286

[4] C R Guilbault, F C Tinsley, Manifolds with non-stable fundamental groups at infinity, III, Geom. Topol. 10 (2006) 541-556 MR2224464

[5] A Hatcher, Algebraic topology, Cambridge Univ. Press (2002) MR1867354

[6] C P Rourke, B J Sanderson, Introduction to piecewise-linear topology, Ergeb. Math. Grenzgeb. 69, Springer, New York (1972) MR0350744

[7] J Stallings, Homology and central series of groups, J. Algebra 2 (1965) 170-181 MR0175956

[8] U Stammbach, Anwendungen der holomogietheorie der gruppen auf zentralreihen und auf invarianten von präsentierungen, Math. Z. 94 (1966) 157-177 MR0201495

[9] C T C Wall, Surgery on compact manifolds, 2nd edition, Mathematical Surveys and Monographs 69, Amer. Math. Soc. (1999) MR1687388 Edited and with a foreword by A A Ranicki

Department of Mathematical Sciences, University of Wisconsin-Milwaukee Milwaukee, WI 53201, USA

Department of Mathematics \& Computer Science, Colorado College

14 East Cache La Poudre St, Colorado Springs, CO 80903, USA

craigg@uwm.edu, ftinsley@ColoradoCollege.edu

https://pantherfile.uwm.edu/craigg/www/, http://faculty1.coloradocollege.edu/ ftinsley/

Received: 11 August $2011 \quad$ Revised: 10 January 2012 